\title{
Simulation studies for optimizing the trigger generation criteria for the TACTIC telescope
}

\author{
M.K. Koul*, A.K. Tickoo, V.K. Dhar, K. Venugopal, K. Chanchalani, \\ R.C. Rannot, K.K.Yadav, P. Chandra, M. Kothari, R. Koul \\ Astrophysical Sciences Division, Bhabha Atomic Research Centre, Mumbai-400085, \\ India.
}

\begin{abstract}
In this paper, we present the results of Monte Carlo simulations of $\gamma$-ray and cosmic ray proton induced extensive air showers as detected by the TACTIC atmospheric Cherenkov imaging telescope for optimizing its trigger field of view and topological trigger generation scheme. The simulation study has been carried out at several zenith angles. The topological trigger generation uses a coincidence of 2 or 3 nearest neighbour pixels for producing an event trigger. The results of this study suggest that a trigger field of $11 \times 11$ pixels $\left(\sim 3.4^{\circ}\right.$ $\left.\times 3.4^{\circ}\right)$ is quite optimum for achieving maximum effective collection area for $\gamma$-rays from a point source. With regard to optimization of topological trigger generation, it is found that both 2 or 3 nearest neighbour pixels yield nearly similar results up to a zenith angle of $25^{\circ}$ with a threshold energy of $\sim 1.5 \mathrm{TeV}$ for $\gamma$-rays. Beyond zenith angle of $25^{\circ}$, the results suggest that a 2-pixel nearest neighbour trigger should be preferred. Comparison of the simulated integral rates has also been made with corresponding measured values for validating the predictions of the Monte Carlo simulations, especially the effective collection area, so that energy spectra of sources (or flux upper limits in case of no detection) can be determined reliably. Reasonably good matching of the measured trigger rates (on the basis of $\sim 207$ hours of data collected with the telescope in NN-2 and NN-3 trigger configurations) with that obtained from simulations reassures that the procedure followed by us in estimating the threshold energy and detection rates is quite reliable.
\end{abstract}

Key words: Monte Carlo simulations; Cherenkov imaging technique; Trigger field of view; Topological trigger; Energy threshold;

PACS: $95.55 . \mathrm{K}$

\footnotetext{
* Corresponding author: mkkoul@barc.gov.in

** Tel: +91-022-25591792 / 25515209
} 


\section{Introduction}

Detection of cosmic $\gamma$-rays in the very high energy range ( $\sim 100 \mathrm{GeV}-50 \mathrm{TeV})$ using ground-based atmospheric Cherenkov technique [1-2] allows a detailed study of the nonthermal universe and probes many phenomena of fundamental astrophysical interest including the acceleration and emission mechanisms in galactic and extragalactic sources, physics of pulsars and supernova remnants, study of extragalactic diffuse background etc. The recent success of the field has mainly resulted from the development of the Cherenkov imaging telescopes which allows efficient separation of photon induced showers from the hadron background. The power of this technique has been convincingly demonstrated by several modern atmospheric Cherenkov telescopes ( e.g. MAGIC [3], VERITAS [4], HESS [5] and CANGAROO [6]) and there is now a general consensus that stereoscopic arrays provide the most promising avenue for future observations.

A typical imaging atmospheric Cherenkov telescope consists of a light collector and a close-packed array of fast photomultiplier tubes ( also called the imaging camera with individual tubes as its pixels). The imaging camera records the spatial distribution of photons in the image (called the Cherenkov image) and by exploiting the differences in the images produced by $\gamma$-ray and cosmic-ray initiated showers, caused by the physical processes responsible for their development in the atmosphere, it becomes possible to effectively segregate the two event species on a statistical basis with a high degree of accuracy. Simulation studies of atmospheric electromagnetic ( $\gamma$-ray induced) and hadronic ( proton induced) cascades $[7,8]$ have shown that there exist subtle differences in the image features ( shape, size and orientation) of these event types. The image of an off-axis $\gamma$-ray shower is relatively more compact and is elliptical in shape, with the major axis of the ellipse oriented towards the camera centre (i.e. the source position). In the case of off-axis cosmic ray shower, on the contrary, the image is comparatively bigger and irregular, besides having no preferred orientation (because of isotropic nature of cosmic rays). Remarkably indeed, these predictions have been thoroughly matched by observations of various groups with great success and it is primarily because of the success of these experiments that the field of ground-based $\gamma$-ray astronomy today boasts a catalog of more than a hundred $\gamma$-ray sources [9].

In an atmospheric Cherenkov imaging telescope, there are several crucial parameters which need to be fine-tuned for maximizing the performance of the system. Assuming that we are observing a point $\gamma$-ray source with the telescope axis directly pointing towards it, these are : (a) the point spread function of the light collector, (b) the pixel size of the imaging camera, (c) trigger field of view and (d) trigger generation scheme. The optimum trigger field of view of an atmospheric Cherenkov imaging telescope needs to be chosen in such a manner that the ratio of $\gamma$-rays to the cosmic ray background is maximum. Thus, for an imaging camera with a large field of view, it is generally advantageous to exclude the outer pixels 
from the trigger generation. These pixels only help to properly reconstruct the image parameters and do not increase the $\gamma$-ray rate. The trigger in an atmospheric Cherenkov imaging telescope is generated by demanding a coincidence between a number of PMTs, with the condition that pixels participating in the trigger should have a signal exceeding some threshold $\mathrm{q}_{0}$. The conventionally used multi-fold ( 2 to 4) coincidence approach necessitates the operation of the telescope at a relatively higher threshold energy in order to keep the chance coincidence rate at a reasonable value. An improved trigger generation scheme involving two or three nearest neighbour pixels can be used instead for lowering the threshold energy of the telescope. The main aim of this work is to use Monte Carlo simulations for optimizing the above mentioned parameters for the TACTIC telescope.

\section{TACTIC imaging telescope}

The TACTIC ( TeV Atmospheric Cherenkov Telescope with Imaging Camera) $\gamma$ ray telescope [10] has been in operation at $\mathrm{Mt}$. Abu $\left(24.6^{\circ} \mathrm{N}, 72.7^{\circ} \mathrm{E}, 1300 \mathrm{~m}\right.$ asl $)$, India, for the last several years to study $\mathrm{TeV}$ gamma ray emission from celestial sources. The telescope uses a tessellated light-collector of area $\sim 9.5 \mathrm{~m}^{2}$ which is capable of tracking a celestial source across the sky. The telescope deploys a 349pixel imaging camera, with a uniform pixel size of $\sim 0.31^{\circ}$ and a $\sim 5.9^{\circ} \times 5.9^{\circ}$ field-of-view, to record atmospheric Cherenkov events produced by an incoming cosmic-ray particle or a $\gamma$-ray photon with an energy above $\sim 1 \mathrm{TeV}$. The TACTIC light-collector uses 34 front-face aluminium-coated, glass spherical mirrors of 60 $\mathrm{cm}$ diameter each with a focal length $\sim 400 \mathrm{~cm}$ and reflection coefficient $>80 \%$ at a wavelength of $\sim 400 \mathrm{~nm}$. The point-spread function has a FWHM of $\sim 0.185^{\circ}$ ( $\equiv 12.5 \mathrm{~mm})$ and $\mathrm{D}_{90} \sim 0.34^{0}$ (三22.8mm). Here, $\mathrm{D}_{90}$ is defined as the diameter of circle, concentric with the centroid of the image, within which $90 \%$ of reflected rays lie. The value of $\mathrm{D}_{90} \sim 0.29^{0}(\equiv 19.3 \mathrm{~mm})$, predicted on the basis of the simulation for an incidence angle of $0^{0}$, matches reasonably well with the measured value mentioned above. Other details regarding the ray-tracing simulation procedure and comparison of the measured point-spread function of the TACTIC light collector with the simulated performance of ideal Davies-Cotton and paraboloid designs are discussed in [11]. The photographs of the TACTIC imaging telescope and its backend signal processing electronics are shown in Fig.1

The imaging camera uses 19mm diameter photomultiplier tubes (ETL-9083 UVB). The bialkali photocathode has a maximum quantum efficiency of $\sim 27 \%$ and for this PMT the maximum quantum efficiency is usually reached in the wavelength range $\sim 340-360 \mathrm{~nm}$. In order to ensure better light collection efficiency and reduction in the background light falling on the photomultipliers we use Compound Parabolic Concentrator (CPC) as light guides on the photomultipliers. The CPCs used in the TACTIC imaging camera have the following specifications : entry aperture $\sim 21.0 \mathrm{~mm}$; exit aperture $\sim 15.0 \mathrm{~mm}$; and height $\sim 17.6 \mathrm{~mm}$. The light col- 
(a)

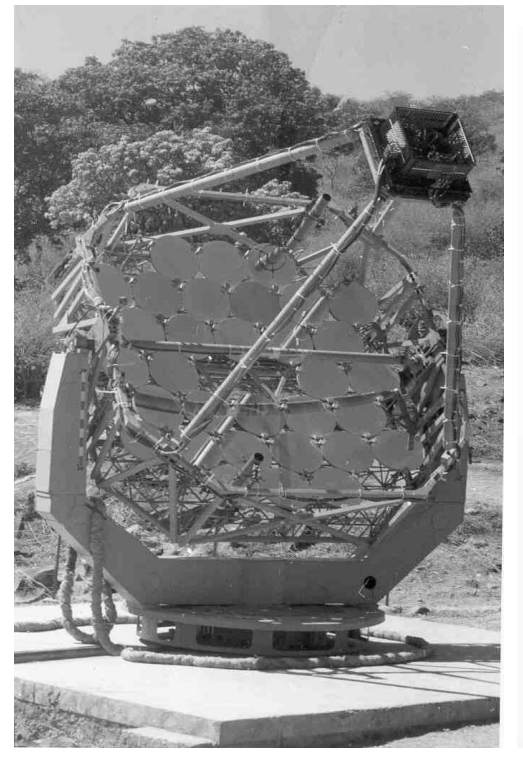

(b)

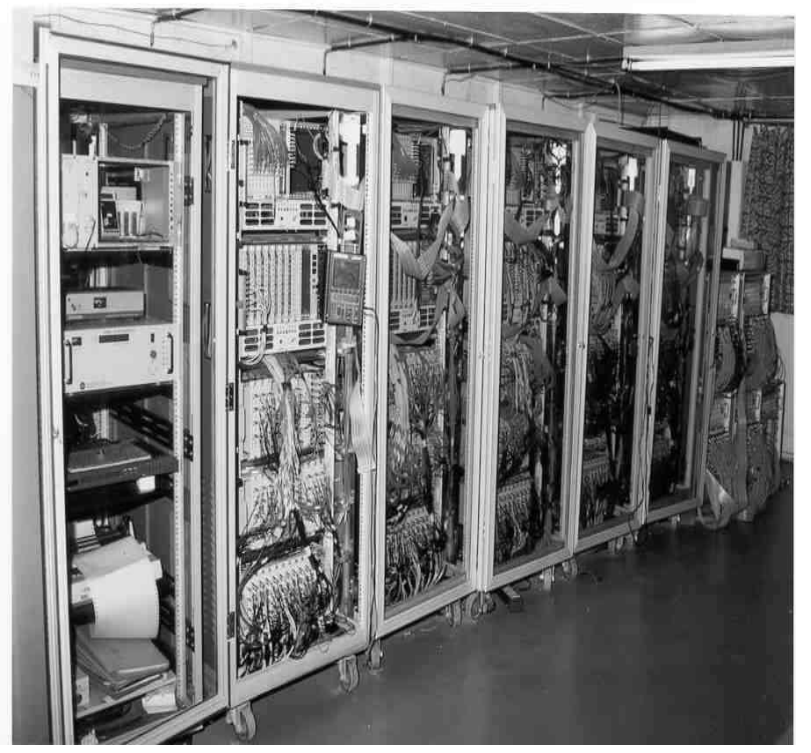

Fig. 1. (a) Photograph of the 349-pixel TACTIC imaging telescope (b) Photograph of back-end signal processing electronics used in the telescope.

lection efficiency of the CPCs is estimated to be of $\sim 65 \%$ by using ray tracing simulations and includes both the geometrical collection efficiency and the reflectivity of the surface. The light collection efficiency mentioned above refers to those photons which undergo reflection after falling on the inside surface of the CPCs. The data acquisition and control system of the telescope [12] has been designed around a network of PCs running the QNX (version 4.25) real-time operating system. The triggered events are digitized by CAMAC based 12-bit Charge to Digital Converters (CDC) which have a full scale range of $600 \mathrm{pC}$. The relative gain of the photomultiplier tubes is monitored by repeatedly flashing a blue LED, placed at a distance of $\sim 1.5 \mathrm{~m}$ from the camera. Whenever the single channel rate of any two or more pixels in the trigger region goes outside the preset operational band, it is automatically restored to within the prescribed range by appropriately adjusting the high voltage of the pixels [13].

The imaging camera uses a programmable topological trigger [14] which can pick up events with a variety of trigger configurations. As the trigger scheme is not hard wired, a number of coincidence trigger options ( e.g Nearest Neighbour Pairs, Nearest Neighbour Non-collinear Triplets and Nearest Neighbour Non-Collinear Quadruplets) can be generated under software control. The number of pixels participating in the trigger can be varied from $7 \times 7$ pixels to $15 \times 16$ pixels. Apart from generating the prompt trigger with a coincidence gate width of $\sim 18 \mathrm{~ns}$, the trigger 
generator has a provision for producing a chance coincidence output based on ${ }^{12} C_{2}$ combinations from various groups of closely spaced 12 channels. It is worth mentioning here that there are several reasons which compel us to use a coincidence gate width of $\sim 18 \mathrm{~ns}$ instead of an optimal gate width $\leq 5 \mathrm{~ns}$. The reasons are the following: (a) variation in the transit time of the PMTs because of being operated at different high voltage values, (b) variation in the propagation delay of 50m long RG58 cables because of procurement of the cables in different batches, (c) degradation in the rise time of the PMT pulses when the signal is transmitted through a $50 \mathrm{~m}$ long RG58 cable and the subsequent 'time-walk' of the leading edge discriminators which is dependent on the amplitude of the PMT output signal and (d) variation in the access time of static RAMS ( Toshiba make TC55B417) used in the trigger generator and difference of $\sim 4 \mathrm{~ns}$ in the cascading and the non-cascading routes [14]. Feasibility of using programmable digital delay generators for fine tuning the signal propagation time in all the channels is also being investigated so that the coincidence gate can be reduced to $<10 \mathrm{~ns}$. Other details regarding the design, implementation and performance evaluation of the programmable topological trigger generator for the 349-pixel imaging camera of the TACTIC telescope are discussed in [14].

A user friendly in-house developed tracking system software provides an independent movement for the zenithal and azimuthal axes so that a matching between the telescope pointing direction and the source direction is obtained with an accuracy better than \pm 2 arc-min. The tracking accuracy of the telescope is also checked on a regular basis with so called 'point runs', where a reasonably bright star, having a declination close to that of the candidate $\gamma$-ray source is tracked continuously for about 5 hours. The point run calibration data (corrected zenith and azimuth angle of the telescope when the star image is centered) are then incorporated in the telescope drive system software so that appropriate corrections can be applied directly in real time while tracking a candidate $\gamma$-ray source. Other relevant details of the instrumentation aspects of the telescope are discussed [10].

\section{Importance of using neighbouring pixels for trigger generation and TAC- TIC trigger generator design}

The conventional approach of generating an event trigger in atmospheric Cherenkov imaging telescopes is to use a majority type coincidence with trigger multiplicity of 2 to 4 . The optimum value of the trigger multiplicity is decided by several factors like observatory altitude, primary energy and zenith angle dependent intensity distribution of the Cherenkov photons in the image plane and the pixel size. However, in order to keep the chance coincidence rate at a reasonable value (usually few percent of prompt coincidence rate), the use of a majority type coincidence approach ( i.e. any $m$ present out of total $n$ detector channels, denoted by any-m,n here onwards ), in generating the trigger, leads to a relatively higher threshold energy. The 
reason for this being the presence of ${ }^{n} C_{m}$ factor in $\mathrm{m}$-fold chance coincidence rate $\left(f_{a n y-m, n}\right)$ formula which is given by

$$
f_{\text {any }-m, n} \approx C_{\text {any-m,n }} m R_{\text {pixel }}\left(q_{0}\right)^{m} \tau_{c}^{m-1}=m \frac{n !}{m !(n-m) !} R_{\text {pixel }}\left(q_{0}\right)^{m} \tau_{c}^{m-1}
$$

where $C_{a n y-m, n}={ }^{n} C_{m}=n ! /(m !(n-m) !)$ is the number of all possible m-pixel combinations, $R_{\text {pixel }}\left(q_{0}\right)$ is the shot-noise generated single channel rate passing the discriminator set at a threshold of $q_{0}$ (in units of photoelectrons), $m$ is the number of detector channels which fire coincidently within a coincidence resolving time $\tau_{c}, n$ is the total number of detector channels which can participate in producing the trigger. It has been assumed in the above formula that the contribution from higher multiplicity triggers (i.e. due to coincidence of $>m$ channels) is negligible as compared to the $\mathrm{m}$-fold chance coincidence rate and this can be satisfied easily as long as $R_{\text {pixel }}\left(q_{0}\right) \tau_{c}<<1$. Keeping in view the compact nature of $\gamma$-ray images, an elegant way to reduce the chance coincidence rate is to limit the number of $m$-fold combinations by using topological constraints in the trigger (such as strict adjacency of triggering pixels or demanding that the pixels should lie within camera sectors). If $C_{N N-m, n}$ is the number of $\mathrm{m}$-fold nearest neighbour combinations the corresponding chance coincidence rate $\left(f_{N N-m, n}\right)$ is given by :

$$
f_{N N-m, n} \approx C_{N N-m, n} m R_{\text {pixel }}\left(q_{0}\right)^{m} \tau_{c}^{m-1}
$$

Since, the number of nearest neighbour m-fold combinations $\left(C_{N N-m, n}\right)$ is always less than the corresponding number of combinations in a simple majority type coincidence $\left(C_{a n y-m, n}\right)$, there is a significant reduction in the chance coincidence rate when nearest neighbour m-fold trigger generation is used. Thus, use of a nearest neighbour $\mathrm{m}$-fold based trigger generation allows the individual pixels to operate at a relatively lower threshold which eventually leads to lowering the threshold energy of the telescope.

An improved trigger generation scheme, based on the above concept was proposed by us [15] for the Whipple system and a reduction in threshold energy by about $20 \%$ is achieved by the group when a 2 adjacent $/ 331$ trigger condition instead of the conventional ${ }^{331} C_{2}$ trigger condition is used [16]. This has resulted in $\sim 40 \%$ increase in the number of $\gamma$-rays detected from the Crab Nebula [16]. The usefulness of the nearest-neighbour trigger generation scheme in achieving the lowest possible energy threshold has been demonstrated by other telescopes also including VERITAS, HESS and MAGIC $[17,18]$. The TACTIC imaging telescope also employs a programmable topological trigger generator with 2( or 3) nearest neighbour pixels participating in the trigger and the main aim of this work is to optimize the overall trigger generation scheme, both with respect to trigger multiplicity (i.e. NN-2 versus NN-3) as well as number of pixels participating in the trigger. Thus, the work involves comparing the performance of NN-2 and NN-3 trigger schemes 
with number of pixels participating in the trigger varying from 49 to 240 .

The number of 2-fold and 3-fold combinations for majority type and NearestNeighbour coincidence for different numbers of pixels participating in the trigger is given in Table. 1 These combinations have been worked out by considering the Table 1

The number of 2-fold and 3-fold combinations for majority and Nearest-Neighbour coincidence trigger schemes.

\begin{tabular}{|c|c|c|c|c|c|}
\hline \hline \multirow{2}{*}{$\begin{array}{c}\text { No. of Trigger } \\
\text { pixels (n) }\end{array}$} & \multirow{2}{*}{$\begin{array}{c}\text { Field of } \\
\text { View }\end{array}$} & \multicolumn{3}{|c|}{ Number of Possible combinations } \\
\cline { 3 - 6 } & & $C_{a n y-2, n}$ & $C_{N N-2, n}$ & $C_{a n y-3, n}$ & $C_{N N-3, n}$ \\
\hline \hline $7 \times 7(=49)$ & $2.17^{\circ} \times 2.17^{\circ}$ & 1176 & 156 & 18424 & 624 \\
\hline $9 \times 9(=81)$ & $2.79^{\circ} \times 2.79^{\circ}$ & 3240 & 272 & 85320 & 1152 \\
\hline $11 \times 11(=121)$ & $3.41^{\circ} \times 3.41^{\circ}$ & 7260 & 420 & 287980 & 1840 \\
\hline $13 \times 13(=169)$ & $4.03^{\circ} \times 4.03^{\circ}$ & 14196 & 600 & 790244 & 2688 \\
\hline $16 \times 15(=240)$ & $4.96^{\circ} \times 4.65^{\circ}$ & 28680 & 869 & 2275280 & 3968 \\
\hline \hline
\end{tabular}

actual layout of the pixels used in the TACTIC imaging camera ( Fig.2a). Different trigger regions with 49,81,121,169 and 240 pixels considered in this work are also indicated in this figure. Defining $\eta_{m, n}=C_{N N-m, n} / C_{a n y-m, n}$ as the reduction in the chance coincidence rate, Fig. $2 \mathrm{~b}$ shows the variation of $\eta_{2, n}$ and $\eta_{3, n}$ as a function of $\mathrm{n}$. With $\eta_{2, n} \sim 4.822 n^{-0.923}$ and $\eta_{3, n} \sim 44.182 n^{-1.843}$, as seen from Fig. $2 \mathrm{~b}$, it is obvious that using a nearest neighbour trigger approach offers an elegant way to reduce the chance coincidence rate. Allowing only certain m-pixel combinations to participate in the trigger also helps in removing a pixels (or certain pixels) with excessive single pixel rate from the trigger without making hardware changes. A very common reason for the excessive single pixel rate is due to presence of relatively bright stars in the field of view of the telescope ( e.g. a star like $\zeta$-Tauri when Crab nebula is being tracked by a telescope).

Regarding the design aspects of the TACTIC trigger generator, while a detailed description of the topological trigger used in the TACTIC telescope can be found in [14], we will only present here some of its key design features. The programmable topological trigger used in the TACTIC telescope is based on 16k x 4 bit fast static RAMs and has a coincidence resolving time of $\sim 18 \mathrm{~ns}$. As the trigger scheme is not hard wired, a number of coincidence trigger options ( e.g NN-2, NN-3 etc.) can be generated under software control. Given the rectangular layout of the TACTIC imaging camera pixels we use a maximum of 240 inner pixels for trigger generation. Since each $16 \mathrm{k}$ x 4 bit RAM can accommodate only 16 pixels, we have divided the trigger pixels into 20 cells of 12 pixels each in the form of a $3 \times 4$ matrix. The outputs from each of the four independently operating trigger generator modules are then collated in an Event Handler module which generates the 22ns 
(a)

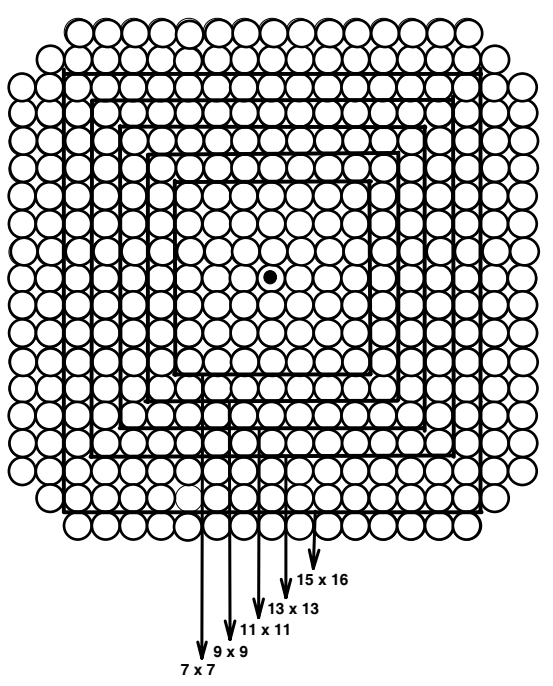

(b)

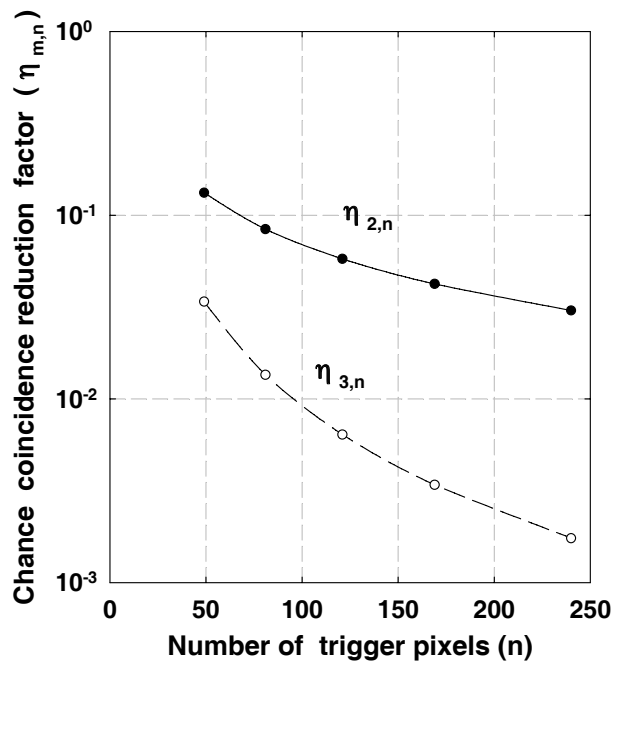

Fig. 2. (a) Layout of the 349-pixels of the TACTIC imaging camera. Different trigger regions with 49,81, 121, 169 and 240 pixels considered in this work are also indicated in the figure. (b) Variation of $\eta_{2, n}$ and $\eta_{3, n}$ as a function of number of pixels in the trigger region.

duration gate pulse and interrupts the Data Acquisition System (DAS) for reading the charge ADC data from all the 349 pixels.

\section{Behaviour of single pixel rate and effect of afterpulsing in PMTs}

It is a very well known fact that the trigger threshold of an atmospheric Cherenkov telescope is dictated by afterpulsing in PMTs instead of fluctuations in the number of photoelectrons due to the light of night sky (LONS) background alone [19,20]. The main aim here is to determine the single pixel threshold in a realistic manner so that the same can be used in Monte Carlo simulations for reliably predicting the telescope performance ( e.g. trigger efficiency as a function of energy, differential rates etc.) when it responds to $\gamma$-ray and cosmic ray showers. The phenomenon of afterpulsing in PMTs leads to generation of large amplitude pulses (well in excess of 10 photoelectrons) at a non-negligible rate. Thus, it is the single pixel rate and the resulting maximum permissible chance coincidence rate which decides the minimal discriminator threshold and ultimately the energy threshold of the telescope. While the phenomenon of afterpulsing (also known as ion feedback) has been investigated thoroughly by a number of workers, we will only discuss here, for the 
sake of completeness, some important consequences of this effect. Afterpulsing is a result of ionization of a molecule or an atom of the residual gas inside the PMT (or adsorption at dynode surface material) by photoelectron and subsequent release of many photoelectrons from the photocathode when the positively charged ion hits the photocathode. The relevance of afterpulses depends on how the PMTs are used and they can largely be inconsequential if the main pulse can be gated. Since the PMTs used in atmospheric Cherenkov imaging cameras are operated in a self-trigger mode (i.e. the PMTs are live all the time), afterpulses pose a serious problem.

Before presenting the measured behaviour of single pixel rate as a function of threshold level, it is worth estimating the single pixel rate $\left(R_{\text {pixel }}\right)$ as a function of the threshold level on the basis of Poissonian fluctuations in the number of photoelectrons. Assuming that the individual LONS photons (and hence the photoelectrons) are uncorrelated and the discriminator threshold is set at $q_{0}$ photoelectrons, $R_{\text {pixel }}\left(q_{0}\right)$ can be estimated by the following formula :

$$
R_{\text {pixel }}\left(q_{0}\right) \approx R_{p e} \frac{\left(R_{p e} \tau_{s}\right)^{q_{0}-1}}{\left(q_{0}-1\right) !} \exp \left(-R_{p e} \tau_{s}\right)
$$

where $\tau_{s}$ is the characteristic shaping time of the single photoelectron response of the PMT ( approximated as a rectangular pulse); $R_{p e}$ is the single photoelectron rate at which photoelectrons are generated; $R_{\text {pixel }}$ is trigger rate such that, within a time $\tau_{s}$, there are $q_{0}$ photoelectrons. It has been also assumed in equation 3 that $R_{\text {pixel }}\left(q_{0}\right) \ll R_{p e}$ and contribution from $>\left(q_{0}\right)$ electrons is negligible to the trigger rate. The single photoelectron rate $\left(R_{p e}\right)$, for a given light intensity, can be obtained by monitoring the average PMT current $\left(I_{a}\right)$ and the PMT gain $\left(G_{P M T}\right)$ and then using the following formula:

$$
R_{p e}=\frac{I_{a}}{e G_{P M T}}
$$

where e is the electron charge. An estimate of the threshold level $\left(\left(q_{0}\right)\right)$, on the basis of the equation 3, can now be readily worked out for a particular telescope system. Using a light of night sky background intensity $\sim 2 \times 10^{12}$ photons $\mathrm{m}^{-2} \mathrm{~s}^{-1} \mathrm{sr}^{-1}$ in the wavelength range $\sim 320-650 \mathrm{~nm}$ for the Mount Abu observatory and other relevant hardware specifications of the TACTIC telescope ( e.g., light collection area, pixel solid angle, quantum efficiency of the PMT etc. ) we find that average number of photoelectrons per $10 \mathrm{~ns}$ time interval is $\sim 0.20$. With a single photoelectron rate of $\sim 20 \mathrm{MHz}$, on using equation 3, it turns out that the PMT trigger rate should be $\sim 1 \mathrm{kHz}$ and $\sim 2.3 \times 10^{-3} \mathrm{~Hz}$ at threshold levels of 5 and 10 photoelectrons, respectively. Actual measurements of the PMT rate as a function of threshold, however, yield much higher values because of afterpulsing. Realizing that the discriminator threshold is a very crucial input required for performing Monte Carlo simulations 
in a realistic manner it becomes necessary to take actual trigger rate measurements. Accordingly, we have also carried out these measurements for several PMTs and the results of this study are discussed below.

For determining the afterpulsing behaviour, laboratory measurements were carried out for several PMTs by simulating the LONS with a LED. The intensity of the LED was set in such a manner, that the resulting PMT anode current is equivalent to the value which the PMT would produce during actual observations. A summary of the results obtained from these measurements is shown in Fig.3. The integral count rate as a function of threshold for 1 PMT using a LONS background $\sim 2.0 \times 10^{12}$ photons $m^{-2} s^{-1} s r^{-1}$ is shown in Fig.3a (marked as $10.3 \mu \mathrm{A}$ ). In order to simulate
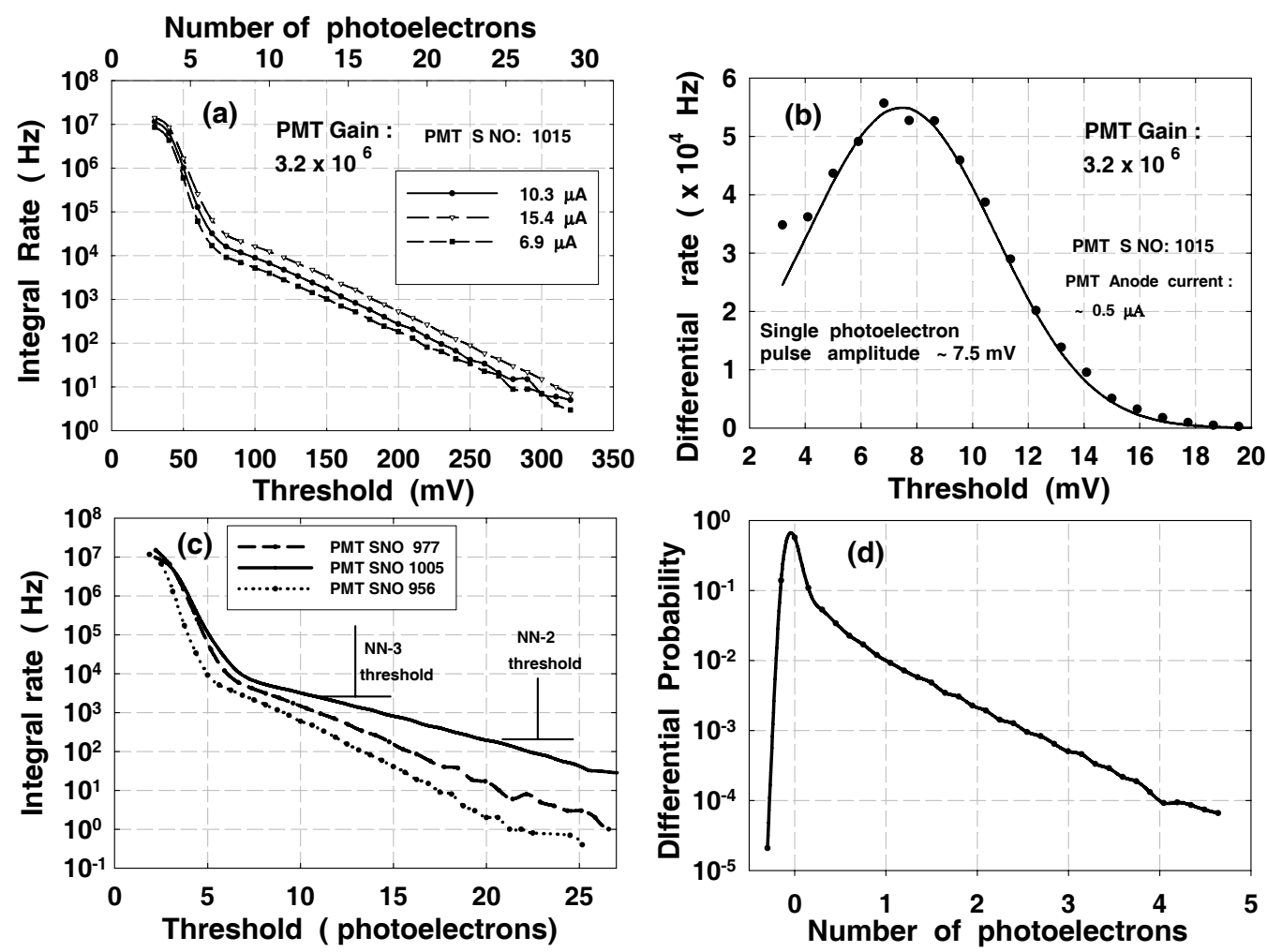

Fig. 3. (a) Variation of trigger rate as a function of discriminator threshold for 3 representative values of light of night sky background values.(b) Differential count as a function of the discriminator threshold for determining the single photoelectron spectrum. (c) Variation of trigger rate as a function of discriminator threshold for 3 different gain calibrated PMTs. (d) Measured amplitude distribution of shot-noise fluctuations in the output of a PMT exposed to a steady light source (like the light of night sky).

the behaviour of integral rate when the telescope is observing a relatively brighter or fainter region in the sky, we also show 2 more representative curves ( marked as $15.4 \mu \mathrm{A}$ and $6.7 \mu \mathrm{A})$ in this figure. The corresponding LONS background values for these two curves has been chosen to be $\sim 3.0 \times 10^{12}$ photons $\mathrm{m}^{-2} \mathrm{~s}^{-1} \mathrm{sr}^{-1}$ and $\sim 1.3 \times 10^{12}$ photons $m^{-2} s^{-1} s r^{-1}$, respectively. It is important to mention here 
that, during actual observations, we operate the PMTs at a lower gain and the typical values of anode currents are $\leq 3 \mu \mathrm{A}$ for most of PMTs. Operation of PMT at higher gain is only followed during laboratory measurements so that the PMT can be calibrated easily. The calibration of the discriminator threshold in terms of photoelectrons (top X-axis scale of Fig.3a ) has been done by determining the gain of the PMT using standard single photoelectron detection method. The measurement for this purpose was taken under very low illumination level and the differential count rate as a function of discriminator threshold is shown in Fig.3b. The integral count rate measurements for 3 additional gain calibrated PMTs is shown in Fig.3c and it is evident from this figure that the afterpulsing rate can vary significantly for the same type of PMT. Although a variation of only $\sim \pm 25 \%$ was reported by us in our earlier work [14] on the basis of about 5 PMTs from the same batch, detailed measurements performed, over last several years indicate the variation in the afterpulsing rate, especially for PMTs which are from different batches, is significantly larger than this value. Factors like variation in the vacuum quality, purity of the dynode material used and the differences in the high voltage needed to reach the same gain can be some of the reasons responsible for this variation. The differences in the afterpulsing rate of PMTs also depends on the threshold level where they are compared. For a large sample of PMTs, procured in different batches, the differences in the afterpulsing rate can be even by a factor of 10 in the threshold level range of $\sim 10-20$ pes. Taking guidance from [21], a function of the type $R_{a p} \sim$ $R_{p e} \times \mathrm{a} \exp \left(-q_{0} b\right)$ ( where $R_{a p}$ is the afterpulsing rate ; a and $\mathrm{b}$ are fit parameters) has also been fit to the afterpulsing rate of the 3 PMTs (Fig. $3 \mathrm{c}$ ) for $\left(q_{0}\right)>5$ pe. The best fit values of $b$ obtained are $\sim 0.29,0.49$ and 0.56 . A typical value of $b \sim 0.5$ has also been reported for the same PMT type by the HEGRA group [21].

Referring back to Fig.3c, one can readily use this plot to determine the discriminator threshold for a predetermined value of PMT trigger rate. For example, taking the case of NN-2 trigger with $C_{N N-m, n}=420$ (i.e. 121 pixels in the trigger region), $\tau_{c} \sim 18 \mathrm{~ns}$ and an additional condition that $f_{N N-m, n} \leq 0.04 \mathrm{~Hz}(\sim 2 \%$ of expected cosmic ray rate of $\sim 2 \mathrm{~Hz}$ ); by using equation 2 we find that $R_{\text {pixel }}\left(q_{0}\right)$ should be in the range of $50 \mathrm{~Hz}$ to $100 \mathrm{~Hz}$ with corresponding values of $q_{0} \sim 22 \mathrm{pe}$. On repeating the above exercise for NN-3 trigger with $C_{N N-m, n}=1840$ it is seen that $R_{\text {pixel }}\left(q_{0}\right)$ should be around $3000 \mathrm{~Hz}$ with a corresponding threshold $q_{0} \sim 13$ pe . Keeping in view that several key telescope parameters (e.g. effective collection area, threshold energy, differential and integral rates) depend on the value $q_{0}$, the above information about $q_{0}$ provides a very important input for carrying out Monte Carlo simulation studies in a realistic manner. The values of $q_{0}$, for the TACTIC telescope with NN-2 and NN-3 trigger configurations have also been marked in Fig.3c . Although one can determine the value of $q_{0}$ by performing Monte Carlo simulations at several trial $q_{0}$ values and eventually choose the one for which the integral rate estimates match the experimental results, following this approach requires intensive computational resources. While we have also followed this approach but the main aim of the exercise was only for the purposes of fine tuning the value of $q_{0}$ and best results were obtained for the following trigger threshold values : $q_{0} \sim 22$ pe for NN-2 trig- 
ger and $q_{0} \sim 14$ pe for NN-3 trigger. We will thus only use these values of $q_{0}$ for rest of the work described in the paper.

In order to carry out realistic simulations for the TACTIC telescope, the simulated data-bases of the photoelectron content of each pixel in a Cherenkov image also needs to include the shot-noise contribution from night sky background light. Fig.3d shows the measured differential pulse amplitude distribution of the shotnoise fluctuations in the output of a PMT. The gate pulse to the charge to digital converter unit for this measurement was generated by using a pulse generator. Using an appropriate probability density function for this noise profile curve, we have then superimposed the effect of shot-noise on the Cherenkov light generated photoelectron content of each pixel in the camera.

\section{Generation of Monte Carlo simulated data base for TACTIC telescope using CORSIKA code}

For the present studies, simulations were carried out using the CORSIKA airshower code (Version 5.6211) [22] with Cherenkov option. The CORSIKA simulation code simulates interactions of nuclei, hadrons, muons, electrons, photons as well as decays of unstable secondaries in the atmosphere. It utilises the EGS4 code for the electromagnetic component of the air shower simulation and dual parton model for the simulation of hadronic interactions. The simulations have been carried out for Mt.Abu observatory altitude of $\sim 1300 \mathrm{~m}$ with appropriate values of $35.86 \mu \mathrm{T}$ and $26.6 \mu \mathrm{T}$ respectively for the horizontal and the vertical components of the terrestial magnetic field.

In order to keep the data-base size and computation time within manageable limits, we have performed multiple sampling of a single shower at several core distances. A total of 104 detector locations (matrix of $52 \times 2$ elements), with each detector having a size of $4 \mathrm{~m} \times 4 \mathrm{~m}$, has been used so that a TACTIC-like telescope can sample Cherenkov light at the core distances of upto $245 \mathrm{~m}$ along one cartesian axis. The position of the shower core has been fixed at the geometrical center of the $52 \times 2$ array. The centre-to-centre inter-element spacing is $10 \mathrm{~m}$ along the $\mathrm{x}$-direction and $6 \mathrm{~m}$ along the $\mathrm{y}$-direction. We have also taken care of the zenith angle dependence on the size of each detector element while performing the simulation at various zenith angles. Again, in order to keep the data-base size within manageable limits, the Cherenkov photon wavelength band chosen for generating the database is $300-450 \mathrm{~nm}$, and the Cherenkov photon bunch size used is varied from 1 to 30 (depending on the energy and arrival direction of the primary particle). The simulated data-base for $\gamma$-ray showers uses about $\sim 38035$ showers in the energy range 0.2 $20 \mathrm{TeV}$ with an impact parameter up to $250 \mathrm{~m}$. These showers have been generated 
Table 2

The number of gamma and proton progenitor showers used in simulation for different values of primary energies at various zenith angles.

\begin{tabular}{|c|c|c|c|c|c|}
\hline \hline \multirow{2}{*}{$\begin{array}{c}\text { Particle Type } \\
\text { Gamma / Proton }\end{array}$} & \multicolumn{5}{|c}{ Number of showers at various zenith angles } \\
\cline { 2 - 6 } & & & & & \\
\hline Energy (TeV) & $5^{\circ}$ & $15^{\circ}$ & $25^{\circ}$ & $35^{\circ}$ & $45^{\circ}$ \\
\hline \hline $0.2 / 0.4$ & $1000 / 1000$ & $1000 / 1000$ & $0 / 0$ & $0 / 0$ & $0 / 0$ \\
\hline $0.3 / 0.6$ & $1000 / 900$ & $1000 / 900$ & $1000 / 1000$ & $0 / 0$ & $0 / 0$ \\
\hline $0.4 / 0.8$ & $1000 / 800$ & $1000 / 800$ & $1000 / 1000$ & $0 / 0$ & $0 / 0$ \\
\hline $0.55 / 1.1$ & $1000 / 750$ & $1000 / 700$ & $1000 / 1000$ & $1000 / 1000$ & $0 / 0$ \\
\hline $0.7 / 1.4$ & $900 / 700$ & $900 / 600$ & $900 / 900$ & $900 / 900$ & $900 / 1000$ \\
\hline $1.0 / 2.0$ & $800 / 650$ & $800 / 550$ & $800 / 800$ & $800 / 800$ & $800 / 900$ \\
\hline $1.3 / 2.6$ & $660 / 600$ & $700 / 500$ & $700 / 700$ & $700 / 700$ & $700 / 800$ \\
\hline $1.8 / 3.6$ & $400 / 550$ & $400 / 450$ & $500 / 600$ & $600 / 600$ & $600 / 700$ \\
\hline $2.5 / 5.0$ & $325 / 500$ & $350 / 400$ & $400 / 500$ & $500 / 500$ & $500 / 600$ \\
\hline $3.3 / 6.6$ & $300 / 450$ & $325 / 350$ & $350 / 450$ & $400 / 450$ & $400 / 550$ \\
\hline $4.5 / 9.0$ & $275 / 400$ & $300 / 300$ & $325 / 425$ & $350 / 425$ & $350 / 500$ \\
\hline $6.0 / 12.0$ & $250 / 350$ & $275 / 250$ & $300 / 400$ & $325 / 400$ & $325 / 450$ \\
\hline $8.0 / 16.0$ & $225 / 300$ & $250 / 200$ & $275 / 375$ & $300 / 375$ & $300 / 400$ \\
\hline $11.0 / 22.0$ & $200 / 275$ & $225 / 175$ & $250 / 350$ & $275 / 350$ & $275 / 350$ \\
\hline $15.0 / 30.0$ & $175 / 250$ & $200 / 150$ & $225 / 325$ & $250 / 325$ & $250 / 300$ \\
\hline $20.0 / 40.0$ & $150 / 225$ & $175 / 125$ & $200 / 300$ & $225 / 300$ & $225 / 250$ \\
\hline \hline Total & $8660 / 8700$ & $8900 / 7450$ & $8225 / 9125$ & $6625 / 7125$ & $5625 / 6800$ \\
\hline \hline
\end{tabular}

at 5 different zenith angles (i.e. $\theta=5^{\circ}, 15^{\circ}, 25^{\circ}, 35^{\circ}$ and $45^{\circ}$ ). Since it is known that the contribution of cosmic ray protons is highest to the total event rate of atmospheric Cherenkov telescopes [23] with other nuclei, including He, contributing up to $\sim 30 \%$ of the total event rate, we have used only proton primaries in our simulation study. Taking guidance from the work [24], where a similar approach has been followed in the simulations, we have scaled up the event rate calculated for protons by a factor of $\sim 1.5$ in order to get a total cosmic ray rate. Accordingly, a data-base for proton showers uses about $\sim 39200$ showers in the energy range 0.4-40 TeV and the incidence angle of these showers is simulated by randomizing the arrival direction of the primary in a field of view of $6.6^{\circ} \times 6.6^{\circ}$ around the pointing direction of the telescope. The number of $\gamma$-ray and proton showers simulated for different primary energies and at various zenith angles are listed in Table 
2. It is important to mention here that the number of gamma-ray showers as well as the number of proton showers have not been generated according to a power law distribution. The main reason for this is to avoid the problem of limited statistics at higher energies on account of too few showers. However, appropriate $\gamma$-ray and proton spectra have been folded in the results while determining differential and integral rates. Similarly, the total number of events(or images) considered for the analysis work is sampled in an off-line manner as per $\sim \mathrm{rdr}$ scaling, where $r$ is the distance of the telescope from the center of the array. The total number of images considered for the present study is $\sim 1.9$ million for $\gamma$-rays and $\sim 2.0$ million for protons.

The CORSIKA code generates a Cherenkov file containing seven parameters for characterizing the Cherenkov photon (or photon bunch) received with a detector element area $(4 \mathrm{~m} \times 4 \mathrm{~m}$ in our case). These parameters are bunch number, $\mathrm{x}$ and $\mathrm{y}$ co-ordinates, two direction cosines, height of production and arrival time at the ground. This CORSIKA code which we have used does not consider wavelength dependent atmospheric absorption of the Cherenkov photon (bunch). Accordingly, atmospheric absorption of the Cherenkov photon-bunch as well as its subsequant history, is taken care of by a supplementary BACUP-code, developed in house. Essential details related of this code are described below.

The parameters of each photon-bunch, produced in the wavelength range, $\lambda \sim 300$ $450 \mathrm{~nm}$ by simulation code CORSIKA and managing to the reach $52 \times 2$ detector array, are written in the Cherenkov file. The BACUP-code uses this information to obtain corresponding parameters of photons expected in the overall wavelength region $\lambda \sim 200-600 \mathrm{~nm}$. Keeping in view the variations in the atmospheric extinction coefficient at shorter and longer wavelength, the step-size used is $5 \mathrm{~nm}, 10 \mathrm{~nm}, 20$ $\mathrm{nm}$ and 50nm for the ranges $\lambda=200-270 \mathrm{~nm}, 270-280 \mathrm{~nm}, 280-400 \mathrm{~nm}$ and $\lambda \geq$ $400 \mathrm{~nm}$ respectively. The number of Cherenkov photons expected in various wavelength intervals is extrapolated using $\sim \lambda^{-2}$ dependence of the Cherenkov light emission spectrum. Each Cherenkov photon is traced back to the point of emission and the $\lambda$ - dependent atmospheric extinction is considered for the wavelength range $\lambda=200-270 \mathrm{~nm}$, and $\lambda \geq 270 \mathrm{~nm}$, using measured data and using data derived from [25] respectively. Each photon-bunch, after correcting for atmospheric absorption, is reflected from the mirror-facet and ray-traced to the corresponding position in the focal plane of the telescope. Appropriate care is taken to include the reflectivity of mirrors, transmission of the light guides (CPC) and quantum efficiency of the phototubes in the present simulation studies. A modest value of $\sim 82 \%$ for the photoelectron collection efficiency has been used in teh simulation. The obstruction encountered due to the telescope mechanical structure by the incident and reflected photon-bunches, during their prorogation, is also duly considered. The data-base of resulting photo-electrons (pe) in the imaging camera pixels, are subjected to noise injection and image cleaning for further analysis. Representative examples of Cherenkov images recorded at a zentih of angle $25^{\circ}$ is shown in Fig. 4. The grey shade color scheme followed, for representing number of photoelectrons recorded 


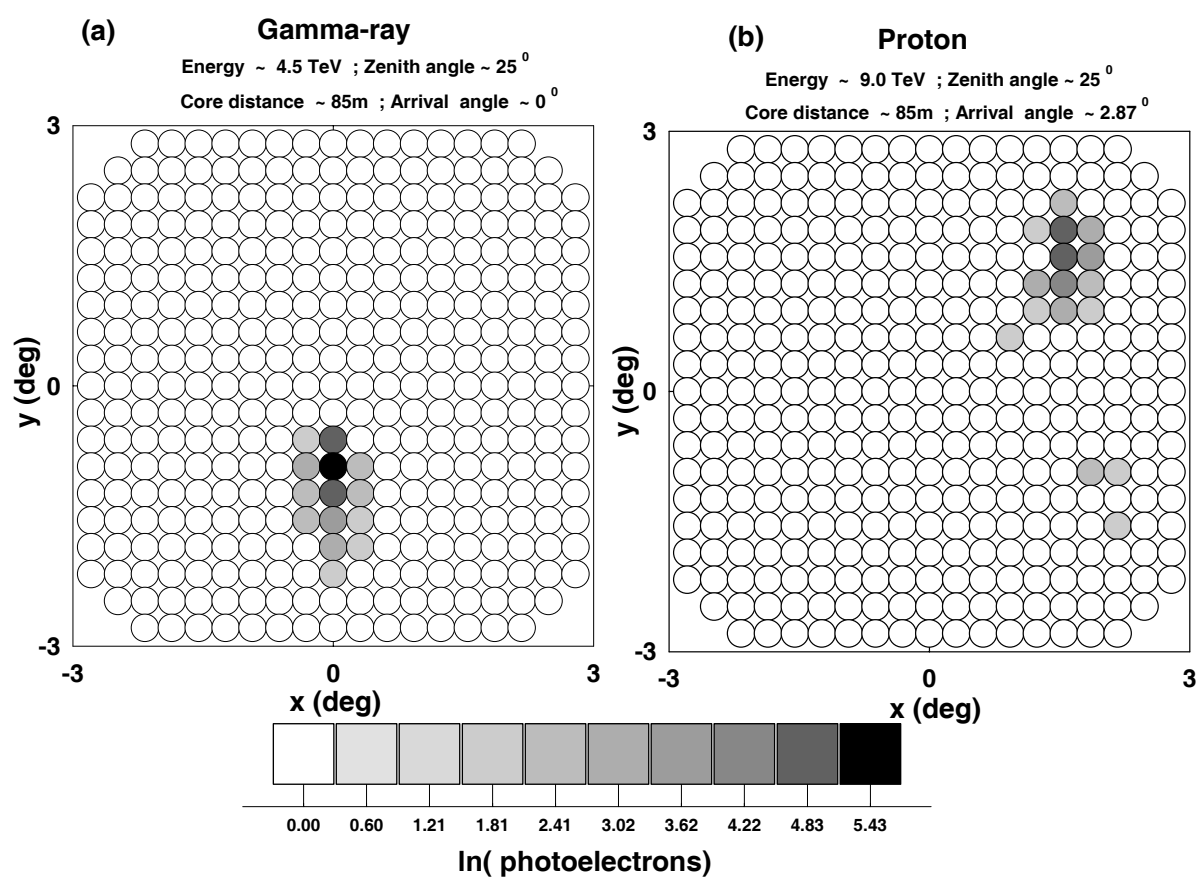

Fig. 4. (a) Cherenkov image of a $\gamma$-ray shower with energy $\sim 4.5 \mathrm{TeV}$ and a core distance of $\sim 85 \mathrm{~m}$. (b) Cherenkov image of a proton shower with energy $\sim 9.0 \mathrm{TeV}$, core distance of $\sim 85 \mathrm{~m}$ and arrival angle $\sim 2.87^{\circ}$.

in a pixel, using equal $\ln$ (photoelectrons) intervals, is also shown at the bottom of this figure. The Cherenkov image of a $\gamma$-ray shower, shown in Fig.4a, has a primary energy of $\sim 4.5 \mathrm{TeV}$ and a core distance of $\sim 85 \mathrm{~m}$. The Cherenkov image, shown in Fig.4b, for an off-axis proton primary has the following parameters : energy $\sim 9.0$ $\mathrm{TeV}$, arrival direction $\sim 2.87^{\circ}$ and core distance of $\sim 85 \mathrm{~m}$.

\section{Formalism for calculating effective collection rate, threshold energy and detection rates}

The trigger rate of an atmospheric Cherenkov imaging telescope and the resulting statistics of the showers is primarily decided by two basic parameters; effective collection area and the energy threshold. For a given configuration of the telescope, these parameters depend on the pixel trigger threshold, the trigger multiplicity and the trigger field of view. The energy threshold of the telescope primarily depends on the geometrical area of the light collector and the photon to electron conversion efficiency. The later being determined by the mirror reflectivity, collection efficiency of the light guides, the spectral response of the PMTs and other possible losses of the Cherenkov light on the way to the PMTs.

The effective collection area of an atmospheric Cherenkov imaging telescope is 
governed by the lateral and the angular distribution of the Cherenkov light generated by the primary particle (i.e. $\gamma$-ray or a cosmic-ray). For a $\gamma$-ray, coming from a point source, the effective collection area $\left(A_{\gamma}(E)\right)$ is given by

$$
A_{\gamma}(E)=2 \pi \int_{0}^{\infty} P_{\gamma}(E, r) r d r
$$

where $P_{\gamma}(E, r)$ is the detection probability (or trigger efficiency) for a $\gamma$-ray with primary energy $\mathrm{E}$ and impact parameter $\mathrm{r}$. By the trigger efficiency $P_{\gamma}(E, r)$, we imply the fraction of the simulated Cherenkov events which can be detected by the telescope placed at a given distance $\mathrm{r}$ from $\gamma$ - ray or proton- generated EAS core. Since, for the isotropic cosmic-ray background the detection probability $P_{C R}(E, r, \Theta)$ depends also on the angle between the direction of the primary particle and the telescope axis $(\Theta)$, the effective collection area $\left(A_{C R}(E)\right)$ is given by

$$
A_{C R}(E)=2 \pi \int_{0}^{2 \pi} \int_{0}^{\infty} P_{C R}(E, r, \Theta) r d r d \Omega
$$

where $d \Omega=2 \pi \sin \Theta$ and the integration over $\Theta$ is included in $d \Omega$. It is important to mention that the effective collection area for cosmic rays, given by equation 6 , has units of $m^{2} s r$. Using the effective collection area derived from above equations, the differential rate can be found by multiplying the effective area with the corresponding differential spectra of $\gamma$-rays and cosmic-rays ; i.e $d R_{\gamma} / d E=A_{\gamma}(E) \times d F_{\gamma} / d E$ and $d R_{C R} / d E=A_{C R}(E) \times d F_{C R} /(d E d \Omega)$. For $\gamma$-rays we have used the Crab Nebula differential spectrum [26]

$$
\left(\frac{d F_{\gamma}}{d E}\right)=6.0 \times 10^{-6} E^{-2.31-0.26 \times \log \left(\frac{E}{300 G e V}\right)} m^{-2} s^{-1} T_{e V}^{-1}
$$

For the cosmic-ray background, we have used the following spectrum [24]

$$
\left(\frac{d F_{C R}}{d E d \Omega}\right)=0.096 E^{-2.7} m^{-2} s^{-1} s r^{-1} T e V^{-1}
$$

As already mentioned in section 5, we only use proton showers for cosmic-ray simulations. The reason for this being the fact that for a given energy, the showers induced by heavier nuclei develop at substantially larger heights in the atmosphere with a less intense Cherenkov light pool. In addition, the fraction of energy channeled into electromagnetic subshowers, responsible for the production of Cherenkov photons decreases with increasing nucleon number. A combination of all these result in a larger but less intense Cherenkov light pool which increases 
the threshold energy of the telescope for heavier cosmic ray particles. The integral trigger rate for $\gamma$-rays $\left(R_{\gamma}\right)$ and cosmic-rays $\left(R_{C R}\right)$ is given by

$$
\begin{aligned}
R_{\gamma}=\int_{0}^{\infty} \frac{d F_{\gamma}}{d E} A_{\gamma}(E) d E \\
R_{C R}=\int_{0}^{\infty} \frac{d F_{C R}}{d E d \Omega} A_{C R}(E) d E
\end{aligned}
$$

It is important to mention here that we have discretized the integrals ( equations 5 and 6) in our simulations with a step size of $10 \mathrm{~m}$ in core distance and a step size of $\sim 0.1^{\circ}$ for the arrival direction. The largest value of core distance used in the simulation is $\sim 245 \mathrm{~m}$ ( imposed by using an array of $490 \mathrm{~m}$ length in the CORSIKA simulations) and this restriction yields reasonably accurate values for primary energy range considered in the present work. Significant deviations in calculation of the effective area are expected at higher primary energies $(>40 \mathrm{TeV})$, but the relative contribution of such particles is only marginal.

\section{Optimization of the trigger field of view}

While pixel size and the total field of view are the basic parameters in the design of a camera for an imaging telescope, it is also important to optimize the trigger field of view so that, $\gamma$-ray showers with an impact parameter of up to $150 \mathrm{~m}$, are able to trigger the telescope. Keeping in view that the detection rate of showers initiated by isotropically distributed cosmic rays is proportional to the solid angle, using a trigger field of view beyond the optimum value only reduces the ratio of $\gamma$-ray trigger rate to cosmic-ray trigger rate. The optimum trigger field of view is decided by the position of the image centroid in the camera and is determined by the height of the shower maximum above the observatory level and the impact parameter. Knowing that, for $\gamma$-ray showers, the angular shift of the image from the camera center can be estimated quite reliably using a simple toy model of the electromagnetic cascade development, it is worth having a look first at these estimates before proceeding to detail Monte Carlo simulations. In the toy model, one assumes that most of the Cherenkov light photons are coming directly from the shower maximum. If $H_{\max }$ is the height of shower maximum (with $\chi_{\max }$ as the corresponding atmospheric depth) where the number of secondary electrons in the shower is maximum, the expression for $\chi_{\max }$, using classical cascade theory is given by :

$$
\chi_{\max } \approx \chi_{0} \ln \left[\left(E_{\gamma} / E_{c}\right)\right]
$$


where $E_{\gamma}$ is the primary energy of the $\gamma$-ray; $E_{c}$ is the critical energy $\left(E_{c} \sim 76\right.$ $\mathrm{MeV})$ and $\chi_{0}$ is the radiation length in the atmosphere $\left(\chi_{0} \sim 36.6 \mathrm{gcm}^{-2}\right)$. The angular displacement of the image centroid from the camera center depends on the impact parameter and the line of sight distance to the shower maximum and is given by the following expression [2]:

$$
\delta_{\text {max }} \approx \tan ^{-1}\left[\frac{b \cos \theta}{b \sin \theta+h_{0} \ln \left[1030 /\left(\chi_{\max } \cos \theta\right)\right]-H_{o b s}}\right]
$$

where $h_{0}$ is the scale height of the atmosphere ( $\left.h_{0} \sim 8.5 \mathrm{~km}\right) ; H_{o b s}$ is the observatory altitude; $b$ is the impact parameter and $\theta$ is the zenith angle. The variation of angular shift of the image centroid as a function of impact parameter for different $\gamma$-ray energies is shown in Fig.5. On examining this figure, it is clearly seen that
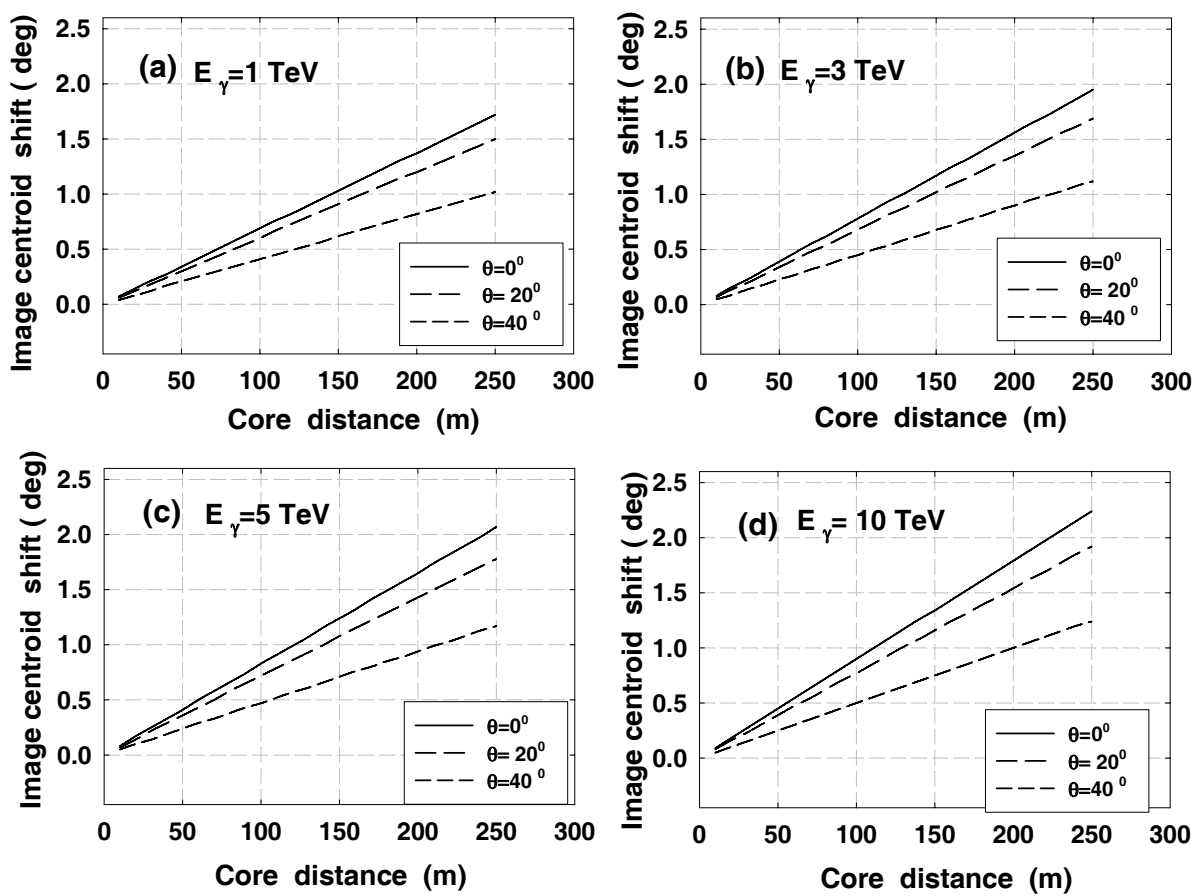

Fig. 5. Angular displacement of the image centroid from the camera center as a function of core distance for various zenith angles $\left(\theta=0^{\circ}, 20^{\circ}\right.$ and $\left.40^{\circ}\right)$ and primary $\gamma$-ray. (a) $E_{\gamma}=1$ $\mathrm{TeV}$ (b) $E_{\gamma}=3 \mathrm{TeV}$ (c) $E_{\gamma}=5 \mathrm{TeV}$ and $E_{\gamma}=10 \mathrm{TeV}$.

an imaging camera with a trigger field of view of $\sim 1.5^{\circ}$ (half-angle) is able to detect $\gamma$-ray showers of energies $<5 \mathrm{TeV}$ up to impact parameter values of $\sim 150 \mathrm{~m}$. It is also seen in the figure that the angular shift of the image centroid from the camera center decreases when zenith angle is increased. Furthermore, if the main concern is to maximize the ratio of $\gamma$-ray trigger rate to cosmic ray trigger rate, further increase in the trigger field of view does not lead to an increase in the $\gamma$-ray trigger rate because of the fact that the detection of $\gamma$-ray showers beyond $\sim 150 \mathrm{~m}$ is limited due to the reduced density of the Cherenkov photons, 
For optimizing the trigger field of view on the basis of Monte Carlo simulations we have used simulated data generated at two zenith angles (viz., $15^{\circ}$ and $35^{\circ}$ ) with trigger regions having 49, 81, 121, 169 and 240 pixels. The corresponding fields of view of these trigger regions are $\sim 2.17^{\circ} \times 2.17^{\circ}, \sim 2.79^{\circ} \times 2.79^{\circ}, \sim 3.41^{\circ} \times$ $3.41^{\circ}, \sim 4.03^{\circ} \times 4.03^{\circ}$ and $\sim 4.65^{\circ} \times 4.96^{\circ}$. Both NN-2 as well as NN-3 trigger generation schemes, with a single pixel threshold $\left(\mathrm{q}_{0}\right)$ of $\sim 14$ pe and $\sim 22$ pe, respectively, have been considered in the simulation study. The trigger efficiency as a function of $\gamma$-ray energy, for showers falling with a core distance of $\sim 250 \mathrm{~m}$ is shown in Fig.6. Referring first to Fig. 6a and Fig. 6b ( i.e efficiency curves at a zenith angle of $15^{\circ}$ for NN-2 and NN-3 trigger modes), it is evident that at $E_{\gamma} \geq 4.0$ $\mathrm{TeV}$, the trigger efficiency increases when the number of pixels in the trigger region is increased from 49 to 121 . It is also seen that the trigger efficiency remains almost constant when the number of trigger pixels is increased further from 121 to 240 . A similar behaviour is also evident in Fig.6c and Fig.6d when a zenith angle of $35^{\circ}$ is used for studying the trigger efficiency. Therefore, these results suggest that using
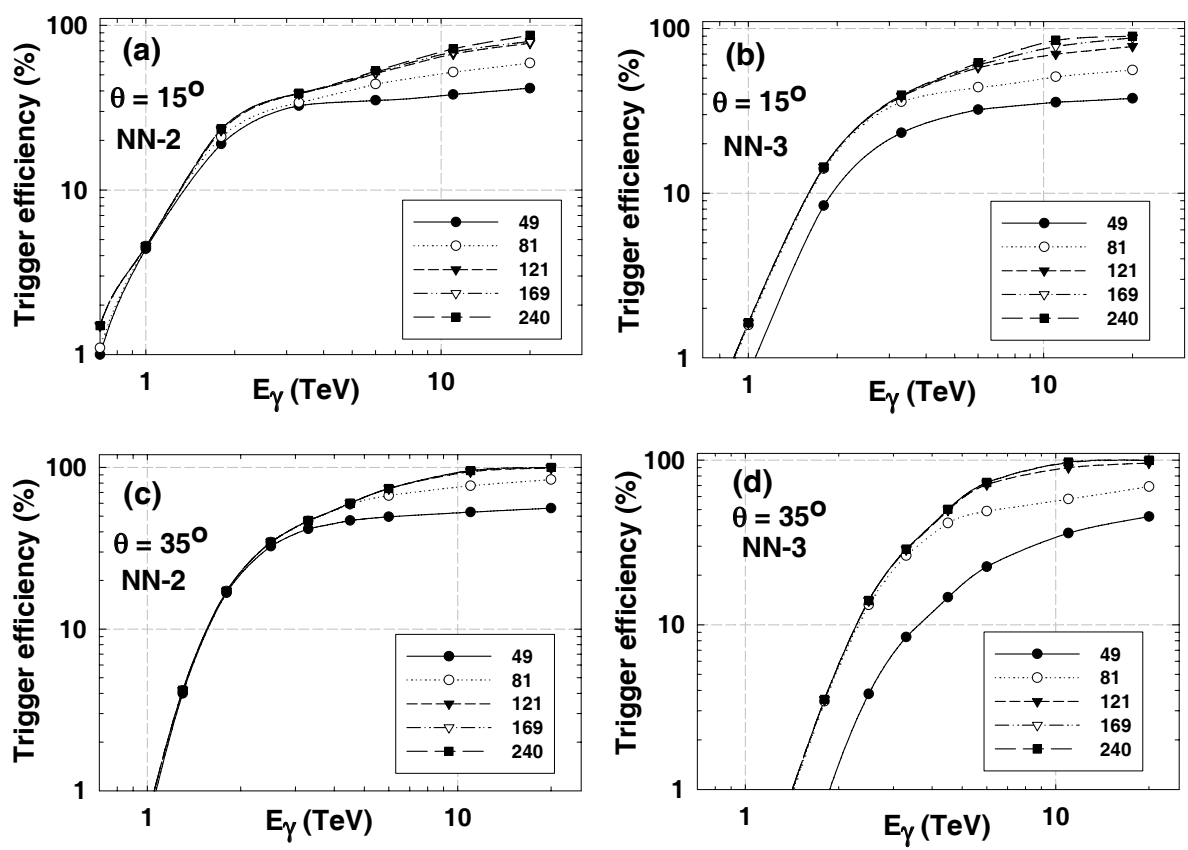

Fig. 6. Trigger efficiency at a function of energy of the primary $\gamma$-rays with trigger regions having 49,81, 121, 169 and 240 pixels. (a) NN-2 trigger with $\theta=15^{\circ}$, (b) NN-3 trigger with $\theta=15^{\circ}$ (c) NN-2 trigger with $\theta=35^{\circ}$ and (d) NN-3 trigger with $\theta=35^{\circ}$.

121 pixels ( $11 \times 11$ matrix), having a field of view of $\sim 3.41^{\circ} \times 3.41^{\circ}$, is quite optimum for producing triggers in response to $\gamma$-rays with an impact parameter up to $\sim 150 \mathrm{~m}$.

We have also estimated the rate loss for cosmic rays when the largest value of core distance used in the simulations is restricted to $\sim 245 \mathrm{~m}$. This restriction arises 
because of using an array of 490m length in the CORSIKA simulations. In this study we have included showers with an impact parameter up to $300 \mathrm{~m}$ and the behaviour of the cosmic ray rate as a function of core distance is shown in Fig.7(ad). Referring first to Fig. 7a and Fig. 7b ( i.e cosmic ray rate at a zenith angle of $15^{\circ}$ for NN-2 and NN-3 trigger modes), one can easily see that, for a trigger field of view with 240 pixels, there is only a marginal increase in the cosmic ray rate ( $\sim 2.5 \%$ ) when the core distance increases from $\sim 245 \mathrm{~m}$ to $\sim 300 \mathrm{~m}$. It is also seen in the figure that corresponding increase in the cosmic ray rate is negligible for a trigger field of view of with 49 pixels. A similar behaviour is also seen in Fig.7c and Fig. $7 \mathrm{~d}$ when a zenith angle of $35^{\circ}$ is used.
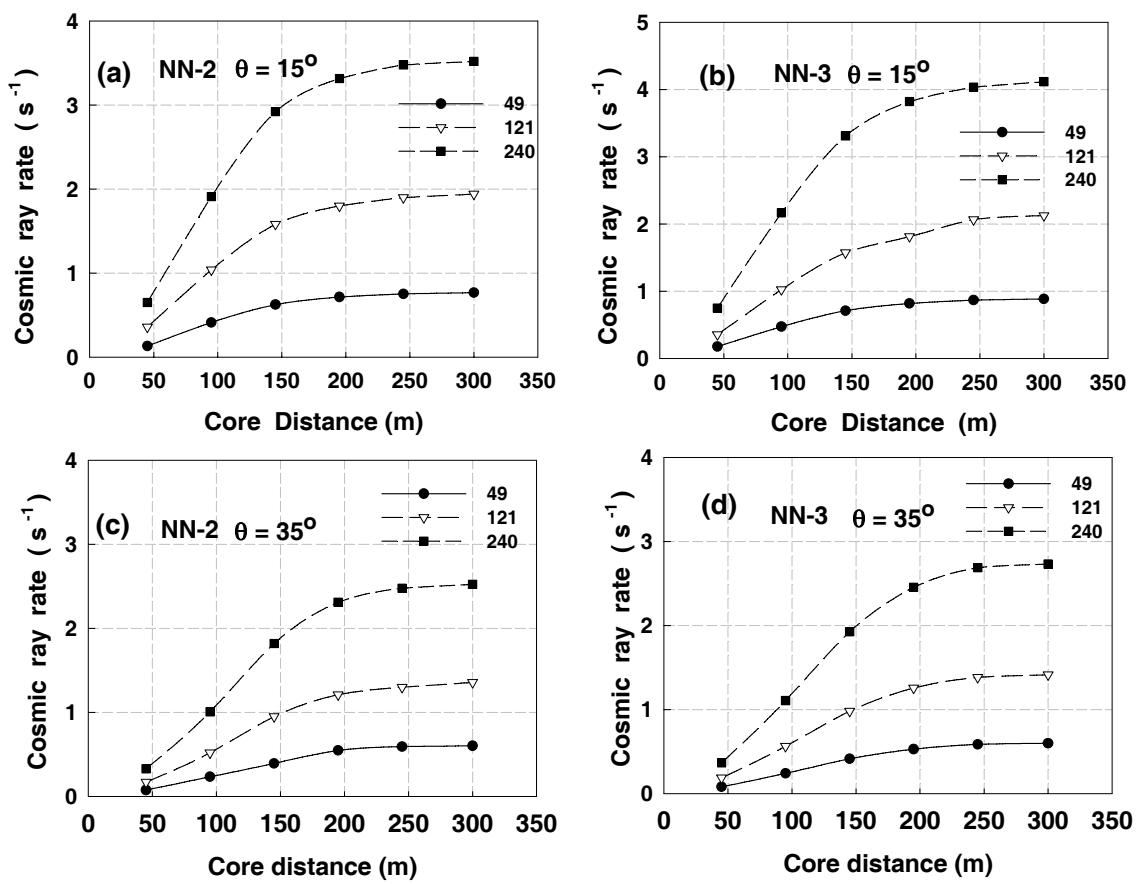

Fig. 7. Cosmic ray rate as a function of core distance with trigger region having 49, 121 and 240 pixels. (a) NN-2 trigger with $\theta=15^{\circ}$, (b) NN-3 trigger with $\theta=15^{\circ}$ (c) NN-2 trigger with $\theta=35^{\circ}$ and (d) NN-3 trigger with $\theta=35^{\circ}$.

The performance of the imaging camera with 49,81, 121, 169 and 240 pixels in the trigger region has also been studied by calculating the integral $\gamma$-ray and cosmic-ray trigger rate and the results of this study are shown in Fig.8. The $\gamma$-ray trigger rates shown in Fig.8a and Fig. $8 b$ have been calculated by using the Crab nebula energy spectrum. The corresponding trigger rates for cosmic-ray protons as a function of trigger field of view are shown in Fig.8c and Fig.8d. Strong dependence of the $\gamma$ ray trigger rate as a function of trigger field of view is only seen when 49 pixels are used for trigger generation. A weak dependence of $\gamma$-ray trigger rate, for all other configurations with $>121$ pixels in the trigger region, is also evident from these figures. With trigger rates of $\sim 40$ events $\mathrm{h}^{-1}$ for $\mathrm{NN}-2$ trigger and $\sim 30$ events 

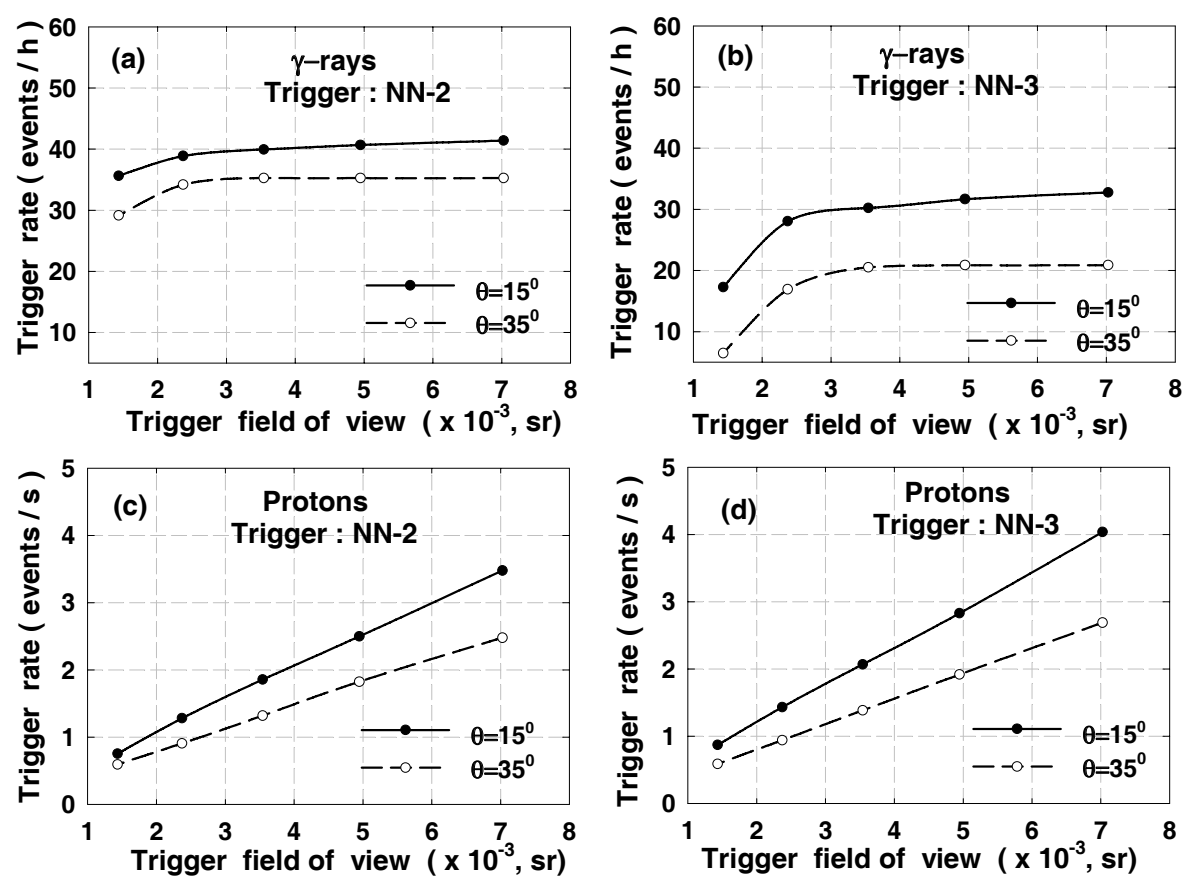

Fig. 8. (a \& b) Gamma-ray trigger rate as a function of trigger field of view for NN-2 and NN-3 trigger schemes. (c \& d) Cosmic-ray trigger rate as a function of trigger field of view for NN-2 and NN-3 trigger schemes.

$\mathrm{h}^{-1}$ for NN-3 trigger at a zenith angle of $15^{\circ}$, it is also seen from these figures that NN-2 trigger scheme provides better detection efficiency at higher zenith angles as compared to the NN-3 trigger scheme. Linear dependence of the proton trigger rate on the trigger field of view seen in Fig.8c and Fig.8d, in accordance with the isotropic behaviour of cosmic rays, also reassures that the trigger field optimization results are reliable. Therefore it can be safely concluded that a trigger region using innermost 121 pixels in the TACTIC imaging camera provides an optimal field of view for detecting $\gamma$-rays from a point source.

It is important to mention here that the actual field of view of the camera should be more than the trigger field so that truncation of the Cherenkov images can be avoided [21]. The shapes of Cherenkov images, produced by $\gamma$-rays, get otherwise distorted and the effect is especially important for the LENGTH parameter (defined as the rms angular size and is related to the longitudinal development of the shower). The limited field of view also introduces large errors in the determination of other image parameters and in estimating the energy of the primary $\gamma$-rays. It is for this reason that imaging camera of the TACTIC telescope uses 225 to 349 pixels. Further work related to realizing the potential of the full camera with 349 pixels, especially using Wobble mode of observation is still in progress. 


\section{Optimization of topological trigger generation scheme on the basis of esti- mated threshold energy and detection rates}

After having validated that a trigger field of view with 121 pixel is quite optimum for detecting the $\gamma$-rays from a point source, we now proceed to performance evaluation of the TACTIC telescope on the basis of detection rates of $\gamma$-rays and cosmic-rays. Optimization of the topological trigger condition by considering 2 and 3 fold nearest neighbour trigger schemes (i.e. NN-2 versus NN-3) is another aspect which is discussed in this section. Collection areas for $\gamma$-ray and cosmic-ray induced showers can be derived from Monte Carlo simulations by evaluating the energy dependent trigger efficiency $\left(P_{\gamma}(E, r)\right.$ and $\left.P_{C R}(E, r, \Theta)\right)$. Using the equations 5 and 6 , along with a single pixel threshold of $\sim 22$ pe for the NN-2 trigger and $\sim 14$ pe for the NN-3, the Monte Carlo evaluated effective collection areas, as a function of the progenitor particle-energy, are shown in Fig.9 for 3-different zenith angles. The results have been obtained by using the inner-most 121 pixels $(11 \times 11$ matrix) for trigger generation. To facilitate an easier comparison of effective ar-
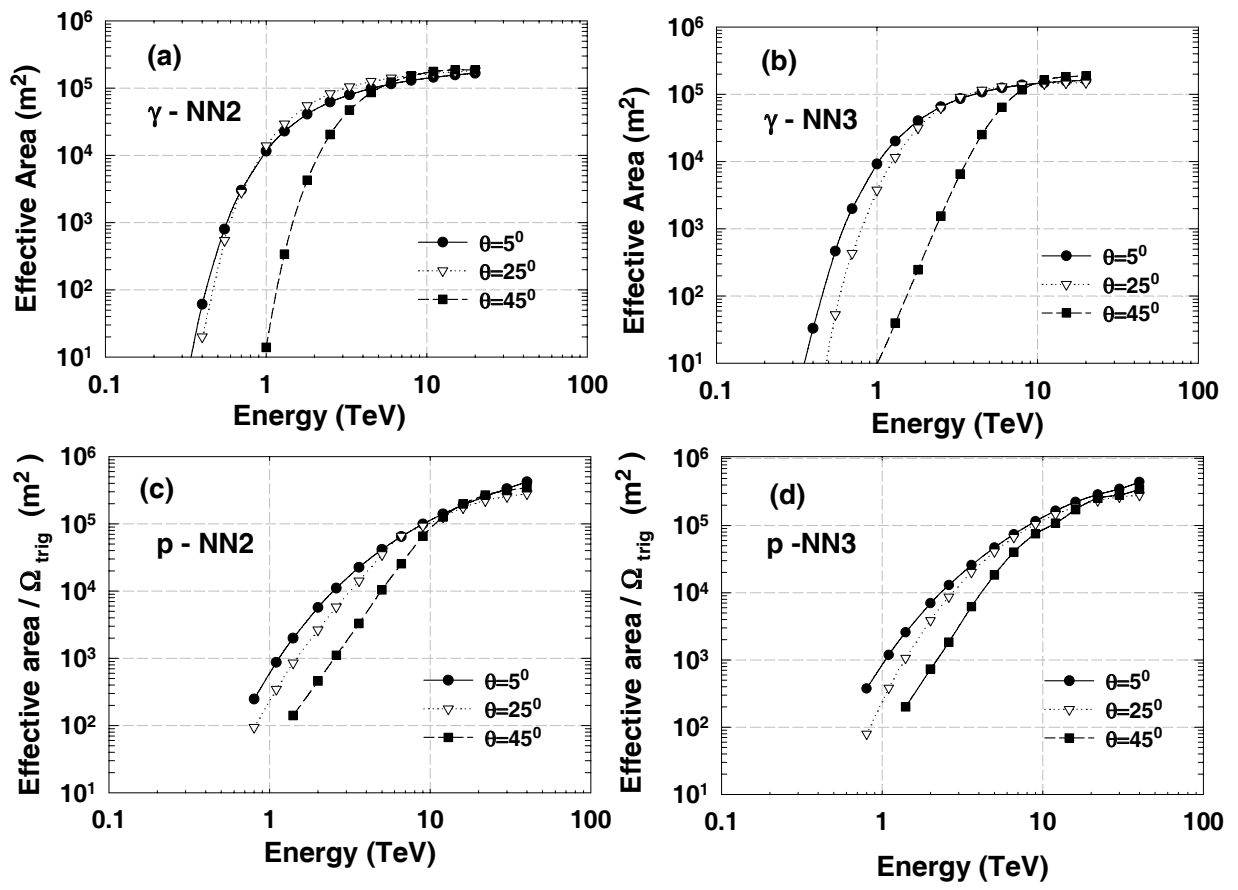

Fig. 9. Effective collection areas for $\gamma$-ray and proton induced showers, at various incident angles, with NN-2 and NN-3 trigger schemes. (a) $\gamma$-rays with NN-2 trigger scheme (b) $\gamma$-rays with NN-3 trigger scheme. (c) Protons with NN-2 trigger scheme.(d) Protons with NN-3 trigger scheme.

eas, we have divided the effective collection area for protons by $\Omega_{\text {trig }}$, where $\Omega_{\text {trig }}$ denotes the trigger field of view solid angle $\left(\sim 3.54 \times 10^{-3} s r\right)$. The energy dependence of the effective collection area on the primary energy is clearly evident in 
this figure and reflects the shape of the lateral distribution of Chrenkov photons on the ground. Compared with the relatively steep lateral distribution of the cosmicray showers, the lateral distribution of Cherenkov light produced by $\gamma$-ray showers has a rather flat lateral distribution up to $\sim 120 \mathrm{~m}$ core distance ( a more precise value is dependent on the observatory altitude). Using derived effective collection areas shown in Fig.9, the corresponding differential rates, for $\gamma$-rays and protons is shown in Fig.10(a-d). The differential rate curves, for the two event species, have
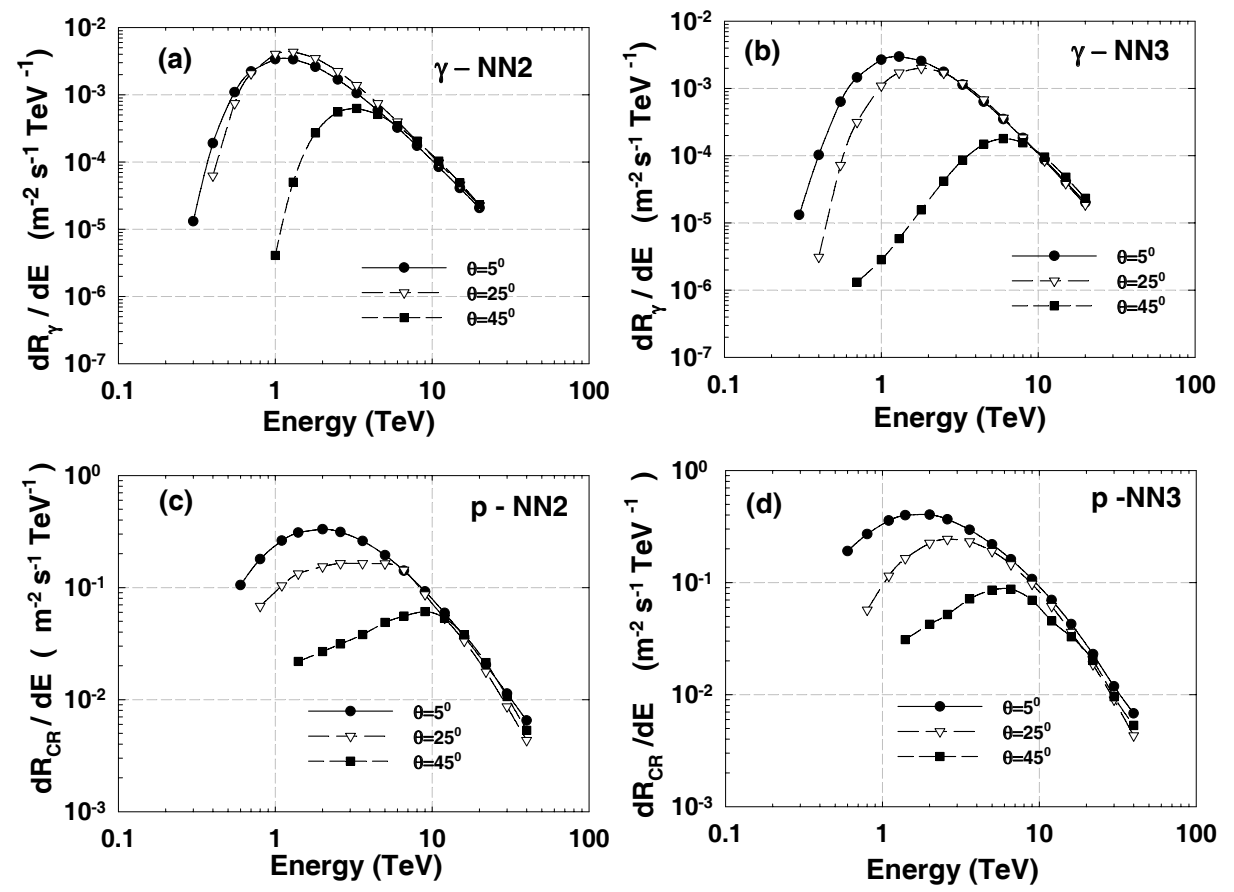

Fig. 10. Estimated differential rates for $\gamma$-ray and proton induced showers, at various incident angles, with NN-2 and NN-3 trigger schemes; (a) $\gamma$-rays with $\mathrm{NN}-2$ trigger scheme (b) $\gamma$-rays with NN-3 trigger scheme; (c) Protons with NN-2 trigger scheme ;(d) Protons with NN-3 trigger scheme.

been obtained by using Crab Nebula spectrum for the $\gamma$-rays (equation 7 ) and proton spectrum ( equation 8). Following the standard definition of effective threshold of the telescope i.e., value of the primary energy at which the differential rate peaks, the resulting energy thresholds of the TACTIC telescope are presented in Table 3. Results regarding integral rates, calculated by using equations 9 and 10, are shown in Fig.11 and also given in Table 3. It is important to mention here that we have scaled up the calculated event rate for protons by a factor of $\sim 1.5$ in order to estimate the total cosmic ray rate ( as discussed in section 5). The main purpose of this scaling is to account for contributions from other nuclei, including $\mathrm{He}$.

On comparing the performance of NN-2 and NN-3 trigger configurations, one can notice the following : (i) In the case of NN-3 trigger, there is a marginal increase in the $\gamma$-ray threshold energy when zenith angle is increased from $5^{\circ}$ to $25^{\circ}$. (ii) 

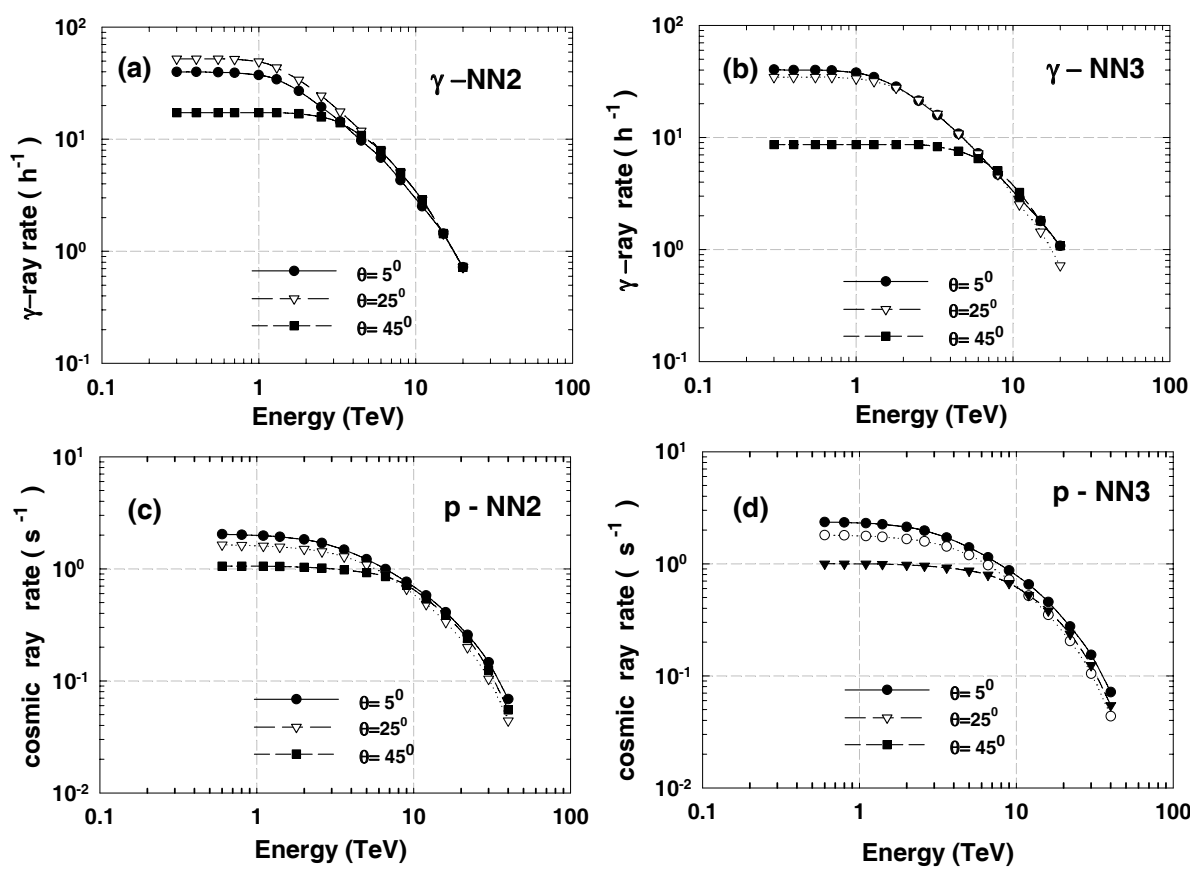

Fig. 11. Estimated integral rates for $\gamma$-ray and cosmic ray induced showers, at various incident angles, with NN-2 and NN-3 trigger schemes. (a) $\gamma$-rays with NN-2 trigger scheme (b) $\gamma$-rays with NN-3 trigger scheme. (c) Cosmic-rays with NN-2 trigger scheme.(d) Cosmic-rays with NN-3 trigger scheme.

Table 3

Threshold energy for $\gamma$-rays (Crab-nebula spectrum) and protons for the TACTIC telescope, with 121 pixels in the trigger region and using NN-2 and NN-3 trigger configurations. The integral detection rates for $\gamma$-rays and cosmic-rays (CR) are also given in the table. A scaling factor of $\sim 1.5$ ( as discussed in section 5) has also been used to estimate the total cosmic-ray rate.

\begin{tabular}{|c|c|c|c|c|c|c|c|c|}
\hline \hline \multirow{2}{*}{$\begin{array}{c}\text { Zenith angle } \\
\text { (in degree) }\end{array}$} & \multicolumn{3}{|c|}{ Energy Threshold (TeV) } & \multicolumn{5}{c|}{ Integral Rate } \\
\cline { 2 - 10 } & \multicolumn{2}{|c|}{$\mathrm{NN}-2$} & \multicolumn{2}{|c|}{$\mathrm{NN}-3$} & \multicolumn{2}{|c|}{$\mathrm{NN}-2$} & \multicolumn{2}{c|}{$\mathrm{NN}-3$} \\
\cline { 2 - 10 } & $\gamma$ & Proton & $\gamma$ & Proton & $\gamma\left(\mathrm{h}^{-1}\right)$ & $\mathrm{CR}\left(\mathrm{s}^{-1}\right)$ & $\gamma\left(\mathrm{h}^{-1}\right)$ & $\mathrm{CR}\left(\mathrm{s}^{-1}\right)$ \\
\hline \hline 5 & 1.3 & 2.6 & 1.3 & 2.6 & 40 & 2.0 & 40 & 2.4 \\
\hline 15 & 1.3 & 3.6 & 1.8 & 3.6 & 36 & 1.9 & 34 & 2.1 \\
\hline 25 & 1.3 & 3.6 & 1.8 & 3.6 & 50 & 1.6 & 34 & 1.8 \\
\hline 35 & 1.8 & 3.6 & 2.5 & 3.6 & 36 & 1.3 & 22 & 1.4 \\
\hline 45 & 3.3 & 9.0 & 6.0 & 9.0 & 18 & 1.0 & 11 & 1.0 \\
\hline
\end{tabular}

Beyond $\theta>25^{\circ}$ the $\gamma$-ray threshold energy increases more rapidly for $\mathrm{NN}$-3 trigger $\left(\sim 6.0 \mathrm{TeV}\right.$ at $\left.45^{\circ}\right)$ as compared to the $\mathrm{NN}-2$ trigger. In the case of NN-2 trigger the $\gamma$-ray threshold energy changes from $1.8 \mathrm{TeV}$ at $\theta=35^{\circ}$ to $\sim 3.3 \mathrm{TeV}$ at $\theta=45^{\circ}$. The 
resulting integral trigger rate for $\gamma$-rays as a function of zenith angle is also seen to drop more rapidly in NN-3 trigger mode compared to the NN-2 trigger configuration. The reason for this being the fact that at larger zenith angles the Cherenkov images produced by $\gamma$-rays tend to be more compact as a result of increase in the line of sight distance to the shower maximum. Using a NN-2 trigger methodology is thus a better option as against the NN-3 trigger configuration when a source needs to be observed at $\theta>30^{\circ}$. Advantage of using a NN-2 trigger configuration, for the HEGRA telescope with a pixel size of $\sim 0.25^{\circ}$, has also been pointed out in [21] while comparing the performance of various nearest neighbour trigger schemes with different multiplicities.

\section{Comparison with experimental data}

The 349-pixel TACTIC imaging telescope, has been in operation at Mt.Abu, India since 2001 and has so far detected $\gamma$-ray emission from the Crab Nebula, Mrk 421 and Mrk 501. First successful detection of $\mathrm{TeV} \gamma$-rays from the Crab Nebula was seen in the data collected by the telescope for $\sim 41.5 \mathrm{~h}$ between January 19 February 23,2001 . The data was taken by using a NN-3 topological trigger with the innermost 240 pixels ( $15 \times 16$ matrix) of the camera participating in generating the event-trigger. A statistically significant excess of $\sim 6.3 \sigma$ was seen in the data with $\sim 447 \pm 71 \gamma$-ray like events. During the last few years, regular observations were taken on a number of potential $\gamma$-ray sources (viz., 1ES2344+514, PSR 0355+54, ON 231, H1426, Mrk-421, Mrk-501 etc.) and the results of these observations have been presented in [27-30]. A major portion of this data has been collected with 121 pixels $(11 \times 11$ matrix $)$ participating in the trigger with either NN-2 or NN-3 trigger configuration and using a total of 225 pixels. At present, the telescope has a $5 \sigma$ sensitivity of detecting Crab Nebula in 25 hours of observation time. This sensitivity figure has been verified several times over last 5 years and consolidated results on the Crab nebula, for $\sim 310.5 \mathrm{~h}$ of observation time taken over a period of 3 observing seasons, is in preparation..

For validating the Monte Carlo simulation results for NN-2 trigger mode we have used data on the Crab Nebula for $\sim 101.44 \mathrm{~h}$ between November 10, 2005 - January 30,2006 . In this data sample, the number of $\gamma$-ray events obtained after applying the Dynamic Supercuts are found to be $\sim(928 \pm 100)$ with a statistical significance of $\sim 9.40 \sigma$ [31]. Similarly, for validating the simulation results for NN-3 trigger mode we have used data on the Crab Nebula for $\sim 105.16 \mathrm{~h}$ between November 03, 2007 - March 8, 2008. After applying the Dynamic Supercuts to this data sample, the number of $\gamma$-ray events are found to be $\sim(968 \pm 90)$ with a statistical significance of $\sim 11.1 \sigma$. Data collected in the NN-2 trigger mode, during November 10, 2005 - January 30, 2006, has also been used by us previously for checking the validity of the ANN-based energy estimation procedure and for determining the energy spectrum of the Crab Nebula in the energy range 1-16 TeV [31]. The 
$\gamma$-ray differential spectrum obtained after using appropriate values of effective collection area and $\gamma$-ray acceptance efficiency (along with their energy and zenith angle dependence) was found to be follow a power law $\left(d \Phi / d E=f_{0} E^{-\Gamma}\right)$ with $f_{0}=(3.12 \pm 0.48) \times 10^{-11} \mathrm{~cm}^{-2} \mathrm{~s}^{-1} \mathrm{TeV}^{-1}$ and $\Gamma=2.69 \pm 0.14$ [31]. While the details of the standard Dynamic Supercuts procedure used by us to separate $\gamma$-ray like images from the background cosmic rays can be found in [27,31], in this work, we will only concentrate on the cosmic ray data collected by the telescope for validating the Monte Carlo simulation estimates regarding effective collection area, threshold energy and integral rates.

Starting with the analysis of the NN-2 trigger mode data collected for $\sim 101.44 \mathrm{~h}$; we have first divided the total data into 4 spells, where each spell corresponds to one lunation period. Without correcting for inter-pixel gain variation and without applying other data selection cuts, Fig.12a shows measured values of cosmic-ray rate as a function of zenith angle. The zenith angle plotted in this figure has been multiplied with the sign of hour angle so that pre-upper transit and post-upper transit rates can be distinguished easily. Each point in this plot represents a 10 minute observation run and the event rate has been calculated by dividing the number of events recorded in 600s. The behaviour of the measured values of cosmic-ray rate as a function of zenith angle for the NN-3 trigger mode is shown in Fig.12b. In this case, the total data has been divided into 5 spells. The corresponding Monte Carlo estimates (solid line), with $R_{C R}(\theta) \sim 2.012(\cos \theta)^{2.054} \mathrm{~Hz}$ for NN-2 trigger and $R_{C R}(\theta) \sim 2.330(\cos \theta)^{2.510} \mathrm{~Hz}$ for NN-3 trigger, are also shown in Fig.12a and Fig.12b, respectively. Although the Monte Carlo estimates are found to describe the zenith angle dependence of the measured rates reasonably well with an accuracy of $\pm \sim 20 \%$ for most of the 10 minute runs, presence of a systematic uncertainty in the experimental data can not be ruled out on account of sky condition variations, both on daily basis as well as from one lunation to other. Differences in parameters like single pixel trigger threshold, overall photoelectron conversion factor etc. are also some of the additional factors because of which there can be a possible mismatch between simulated trigger rates and the corresponding experimental values. While work on understanding the telescope systematics is still in progress, we have used a value $\sim 15 \%$ for systematic error in the measured cosmic-ray rate for further analysis. The justification for this is based on the guidance from [32] where the author has reported a variation of $\sim 15 \%-20 \%$ in the Chrenkov photon density for mid-latitude sites on account of seasonal changes. Although, one does not expect a significant variation in atmospheric extinction on a nightly basis for a good astronomical site, significant variation in atmospheric extinction on a nightly basis at Mt. Abu has been observed by us over the last several years. Using a value $\sim 15 \%$ for systematic error in the measured cosmic-rate is thus a fairly conservative estimate.

The measured cosmic-ray rates have also been normalized to a zenith angle of $0^{\circ}$ and variation of the normalized rates as a function of run number is shown in Fig.12c (NN-2 trigger ) and Fig.12d (NN-3 trigger). In order to quantify departures from a flat shape, we have also calculated the $\chi^{2}$ by using the following expression 

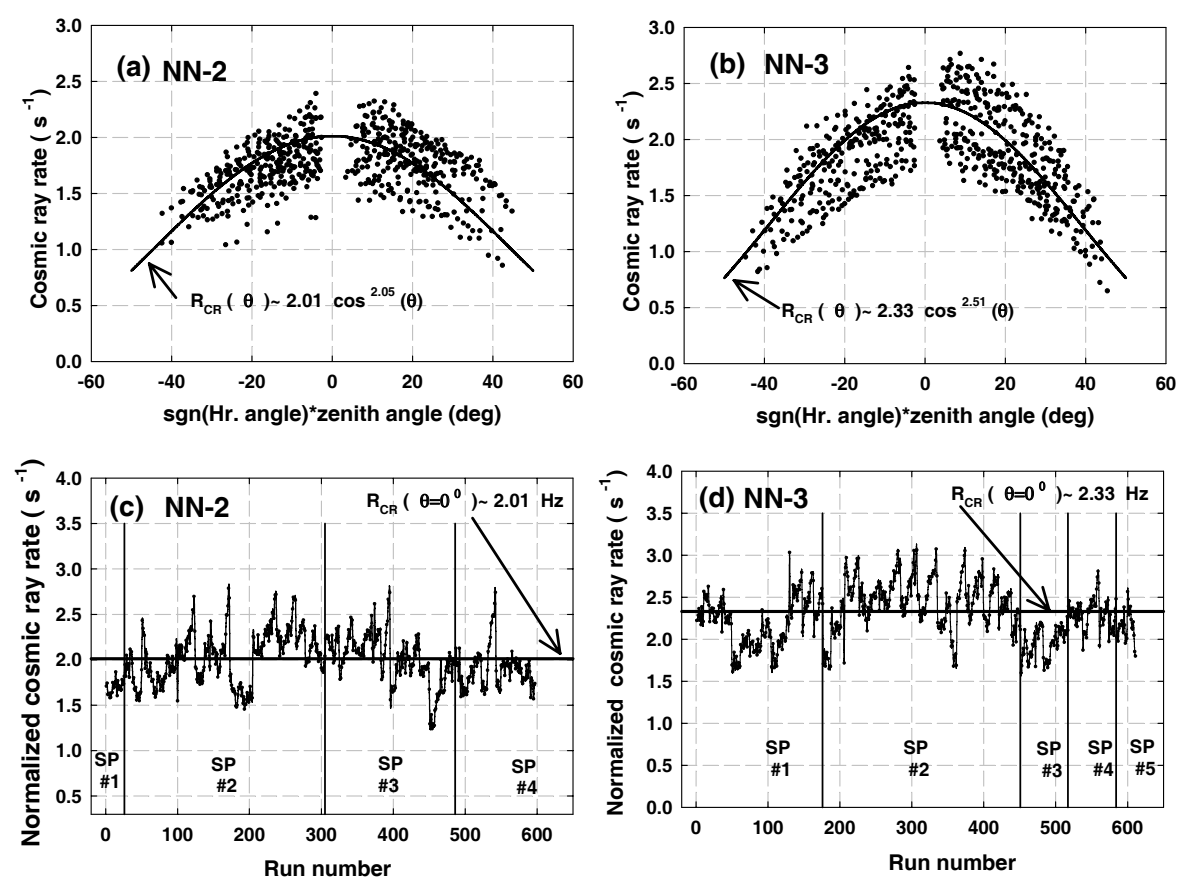

Fig. 12. Measured cosmic ray trigger rate as a function of zenith angle (a) data collected between November 10, 2005 - January 30, 2006 with NN-2 trigger configuration;(b) data collected between November 03, 2007 - March 8, 2008 with NN-3 trigger configuration. The spline curve shown in theses figure is the Monte Carlo-based estimated cosmic-ray trigger rate. Normalized cosmic ray rate as a function of run number (c) November 2005 - January 2006 data with NN-2 trigger (d) November 2007 - March 2008 data with NN-3 trigger. The segmentation of the data into various spells are indicated by full vertical lines. Each spell corresponds to one lunation period and the notation SP\# stands for spell number.

[33]

$$
\chi^{2}=\sum_{i=1}^{n_{\text {run }}} \frac{\left(R_{C R, i}-R_{C R}^{M C}\right)^{2}}{\left(\Delta R_{C R, i}\right)^{2}+\left(f_{i}^{r e l} R_{C R, i}\right)^{2}}
$$

where $n_{\text {run }}$ is the number of 10 minute runs, $R_{C R, i}$ is the normalized measured cosmic-ray rate with $\Delta R_{C R, i}$ as the corresponding statistical error, $f_{i}^{r e l}$ is the relative systematic uncertainty and $R_{C R}^{M C}$ is the normalized Monte Carlo simulated trigger rate. The resulting values of $\chi^{2} / d o f \sim 605.4 / 597$ (probability $=0.40$ ) obtained for the NN-2 trigger observation spell and $\chi^{2} / d o f \sim 662.6 / 611$ (probability $=0.07$ ) obtained for the NN-3 trigger observation spell clearly suggest that there is a reasonably good matching between the simulated trigger rates and the experimentally measured trigger rates. Presence of a systematic error at $>15 \%$ level for November 2007 - March 2008 data may also be contributing to the relatively higher value of $\chi^{2}$ for this data spell.

The homogeneity of the triggers produced within the trigger field of view is another 
test to check the performance of the trigger generation scheme. Although finding the frequency distribution of the triggered pixels would have been the best way to validate this, unavailability of the hit pattern data at the hardware level does not allow us to check this directly. To circumvent this, we determine the frequency distribution of the image centroid coordinates by using the following approach. First, the data is corrected for inter-pixel gain variation and then subjected to the standard two-level image 'cleaning' procedure [27] with picture and boundary thresholds of $6.5 \sigma$ and $3.0 \sigma$ respectively. In the clean Cherenkov image, if $i t h$ PMT with coordinates $x_{i}, y_{i}$ registers a signal $s_{i}$, the coordinates of the image centroid are given by : $<x>=\sum_{i} s_{i} x_{i} / \sum_{i} s_{i}$ and $\langle y\rangle=\sum_{i} s_{i} y_{i} / \sum_{i} s_{i}$. Considering the central $\sim 2.25^{\circ}$ $\times 2.25^{\circ}$ trigger region and dividing it into 225 cells of size $\sim 0.15^{\circ} \times 0.15^{\circ}$ each, we bin the image centroid co-ordinates into these angular cells. Assuming that the image centroid is very close to the brightest pixel (i.e. pixel with highest signal), the resulting centroid map can be faithfully used for checking the homogeneity of the triggers produced within the trigger field of view. Defining $N_{c e l l, i}$ as number of events in the ith cell and $\left\langle N_{\text {cell }}\right\rangle$ as the average number of events in each cell we calculate percentage residual of events $\left(\Delta N_{\text {cell }, i}\right)$ in each cell where $\Delta N_{\text {cell }, i}$ $\left.=\left(N_{\text {cell }, i}-\left\langle N_{\text {cell }}\right\rangle\right) /<N_{\text {cell }}\right\rangle$. In order to ensure that images are robust and are contained within the camera we have also used the following cuts for selecting the events for this analysis $:|<x>|<1.2^{\circ},|<y>|<1.2^{\circ}$, SIZE $\geq 450$ d.c where (6.5 digital counts $\equiv 1.0$ pe $)$ and number of pixels participating in the image $\geq 4$. Fig.13 shows the results of the percentage residual events $\left(\Delta N_{c e l l, i}\right)$ for the NN-2 and NN-3 observation spells. A gray shade coding scheme has been used in this figure for representing the percentage residual events. The corresponding results obtained for the NN-3 trigger configuration by using data collected during November 03, 2007 - March 8, 2008 is shown in Fig.13b. In the NN-2 data ( Fig.13a) we find that $\sim 69 \%$ of the cells have $\left|\Delta N_{\text {cell }, i}\right|$ with in $\sim 20 \%$. The corresponding number of cells, for the NN-3 data ( Fig.13b), is found to be $\sim 65 \%$. Presence of non-functional pixels in the camera (typically in the range of 3 to 7 in a total of 225 pixels and whose location keeps on changing with time) as well as excessive trigger rate in some pixels due to presence of relatively bright stars are the 2 main causes because of which $\left|\Delta N_{\text {cell,i }}\right|$ is more than $\sim 30 \%$ in some regions of the camera.

Compatibility of the trigger rates with Poissonian statistics and homogeneity of the triggers produced within the trigger field of view clearly suggest that the overall performance of the telescope is quite stable and the Monte Carlo simulation estimates regarding effective collection area and $\gamma$-ray acceptance are also quite reliable. The agreement between the expected and actual performance of the telescope has also been checked by comparing the expected and observed image parameter distributions and results of this study can be seen in [10]. The consistent detection of a steady signal from the Crab Nebula above $\sim 1.2 \mathrm{TeV}$ energy, at a sensitivity level of $\sim 5.0 \sigma$ in $\sim 25 \mathrm{~h}$, alongwith excellent matching of its energy spectrum with that obtained by other groups, also reassures that the performance of the TACTIC telescope is quite stable and reliable [27,29,31]. However, more work has to be 

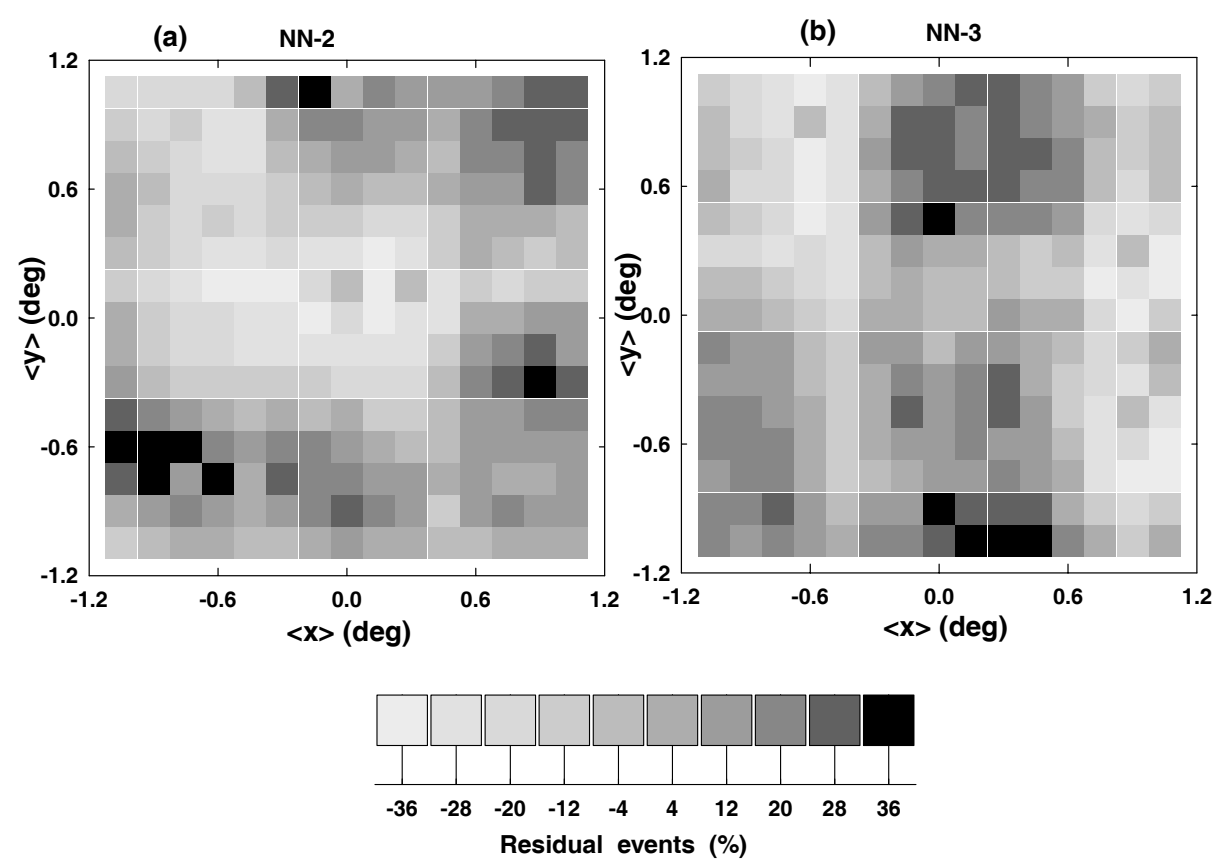

Fig. 13. (a) Percentage residual events ( $\Delta N_{c e l l, i}$ ), for the NN-2 data, on the basis of the image centroid coordinates by following gray shade coding scheme. (b) Same as (a) except with NN-3 trigger configuration. The gray shade coding scheme followed for representing the percentage residual events is also given at the bottom of this figure.

done with regard to relative calibration of the telescope ( along the lines suggested in [34]) so that unavoidable systematic errors as a result of temporal changes in the light collection efficiency, gain and, most importantly, atmospheric conditions can be accounted for in the analysis.

\section{Conclusions}

A detailed simulation study for the TACTIC telescope has been presented in this work for optimizing its trigger field of view and the trigger generation scheme. Different trigger regions with 49,81,121,169 and 240 pixels were considered in this work and the results suggest that a trigger field of $11 \times 11\left(\sim 3.4^{\circ} \times 3.4^{\circ}\right)$ is quite optimum for achieving maximum detection rate for $\gamma$-rays from a point source. With regard to optimization of topological trigger generation, it is found that using a NN-2 trigger methodology is a better option as against the NN-3 trigger configuration when a source needs to be observed at zenith angles of $>25^{\circ}$. Reasonably good matching of the measured trigger rates (on the basis of $\sim 207$ hours of data collected with the telescope in NN-2 and NN-3 trigger configurations) with that obtained from simulations reassures that the procedure followed by us in estimat- 
ing the threshold energy and detection rates is quite reliable. Furthermore, excellent matching of Crab Nebula differential spectrum with that obtained by other groups, reported by us in our earlier work, also reassures that the procedure followed by us for obtaining the energy spectrum of a $\gamma$-ray source is quite reliable. Attempts are also being made at present to improve the sensitivity of the telescope. Apart from using new CPCs ( modified CPC with square entry and a circular exit) for enhancing the light collection efficiency of the camera, we are also trying to reduce the coincidence gate width. Both these modifications are expected to lower the threshold energy of the TACTIC telescope. To conclude, we firmly believe that there is considerable scope for a TACTIC like imaging telescope for monitoring AGNs on a long term basis.

\section{Acknowledgements}

The authors would like to convey their gratitude to all the concerned colleagues of the Astrophysical Sciences Division for their contributions towards the instrumentation and observation aspects of the TACTIC telescope. We would also like to thank the anonymous referee for his valuable comments and suggestions which have helped us to improve the quality of the paper.

\section{References}

[1] P.M. Chadwick, I.J.Latham, S.J.Nolan, J.Phys. G : Nucl. Part. Phys., 35 (2008) 033201.

[2] F. Aharonian et al., Rep. Prog. Phys., 71 (2008) 096901.

[3] E. Lorentz et al., New Astron. Rev., 48 (2004) 339.

[4] T.C. Weekes et al., Astropart. Phys., 17 (2002) 221.

[5] J.A. Hinton et al., New Astron. Rev., 48 (2004) 331.

[6] H. Kubo et al., New Astron. Rev., 48 (2004) 323.

[7] V.I. Zatsepin, Soviet Phys. JETP-USSR, 20 (1965) 459

[8] A.M. Hillas, Proc. 19th ICRC, La Jolla, 3 (1985) 445.

[9] http://www.mppmu.mpg.de/ rwagner/sources/index.html

[10] R. Koul et al., Nucl. Instr. and Meth. A, 578 (2007) 548.

[11] A.K. Tickoo et al., Nucl. Instr. and Meth. A, 539 (2005) 177.

[12] K.K. Yadav et al., Nucl. Instr. and Meth. A, 527( 2004) 411. 
[13] N. Bhatt et al., Meas. Sci. Technol., 12 (2001) 167.

[14] S.R. Kaul et al., Nucl. Instr. and Meth. A, 496 (2003) 400.

[15] C.L. Bhat et al., Nucl. Instr. and Meth. A, 340 (1994) 413.

[16] S.M. Bradbury and H.J.Rose, Nucl. Instr. and Meth. A, 481 (2002) 521.

[17] S. Funk et al., Astropart. Phys., 22 (2004) 285.

[18] D. Bastieri et al., Nucl. Instr. and Meth. A, 461 (2001) 521.

[19] R. Mirzoyan et al., Proc. Towards a Major Atmospheric Cherenkov Detector -IV, Padova, Italy, 1995, p.230

[20] R. Mirzoyan et al., Nucl. Instr. and Meth. A, 387 (1997) 74.

[21] C. Kohler et al., Astropart. Phys., 6 (1996) 77.

[22] D. Heck et al., Report FZKA 6019 Forshungszentrum, Karlshrue, (1998).

[23] F. Aharonian et al., Phys. Rev. D, 59 (1999) 092003.

[24] A. Konopelko, Astropart. Phys., 24 (2005) 191.

[25] L. Eltermann, 1968, AFCRL 68-0153 Air Force Cambridge Res. Lab., Massachuesett.

[26] J. Albert et al., Ap J., 674 (2008)1037.

[27] K.K. Yadav et al., Astropart. Phys., 27 (2007) 447.

[28] S.V. Godambe et al., J.Phys. G : Nucl. Part. Phys., 34 (2007) 1683.

[29] S.V. Godambe et al., J.Phys. G : Nucl. Part. Phys., 35 (2008) 065202.

[30] K.K. Yadav et al., J.Phys. G : Nucl. Part. Phys., 36 (2009) 085201.

[31] V.K. Dhar et al., Nucl. Instr. and Meth. A, 606 (2009) 795

[32] K. Bernlohr et al., Astropart. Phys., 12 (2000) 255.

[33] A.A. Abdo et al., ApJS, 188 (2010) 405.

[34] S. LeBohec and J.Holder, Astropart. Phys., 19 (2003) 221. 